\title{
HOMOGENIZATION OF QUASI-CRYSTALLINE FUNCTIONALS VIA TWO-SCALE-CUT-AND-PROJECT CONVERGENCE*
}

\author{
RITA FERREIRA ${ }^{\dagger}$, IRENE FONSECA $^{\ddagger}$, AND RAGHAVENDRA VENKATRAMAN ${ }^{\ddagger}$
}

\begin{abstract}
We consider a homogenization problem associated with quasi-crystalline multiple integrals of the form $u_{\varepsilon} \in L^{p}\left(\Omega ; \mathbb{R}^{d}\right) \mapsto \int_{\Omega} f_{R}\left(x, \frac{x}{\varepsilon}, u_{\varepsilon}(x)\right) d x$, where $u_{\varepsilon}$ is subject to constantcoefficient linear partial differential constraints. The quasi-crystalline structure of the underlying composite is encoded in the dependence on the second variable of the Lagrangian, $f_{R}$, and is modeled via the cut-and-project scheme that interprets the heterogeneous microstructure to be homogenized as an irrational subspace of a higher-dimensional space. A key step in our analysis is the characterization of the quasi-crystalline two-scale limits of sequences of the vector fields $u_{\varepsilon}$ that are in the kernel of a given constant-coefficient linear partial differential operator, $\mathcal{A}$, that is, $\mathcal{A} u_{\varepsilon}=0$. Our results provide a generalization of related ones in the literature concerning the $\mathcal{A}=$ curl case to more general differential operators $\mathcal{A}$ with constant coefficients and without coercivity assumptions on the Lagrangian $f_{R}$.
\end{abstract}

Key words. homogenization, quasi-crystalline composites, multiscale variational problems, PDE constraints, two-scale-cut-and-project convergence, $\Gamma$-convergence

AMS subject classifications. 49J45, 35E99

DOI. $10.1137 / 20 \mathrm{M} 1341222$

1. Introduction. The theory of homogenization addresses the description of the macroscopic or effective behavior of a microscopically heterogeneous system. There are multiple applications in the fields of physics, mechanics, and materials science and in other areas of engineering, including problems aimed at the modeling of composites, stratified or porous media, finely damaged materials, or materials with many holes or cracks.

From the mathematical viewpoint, homogenization is often associated with the study of the asymptotic behavior of oscillating partial differential equations (PDEs), or of minimization problems deriving from certain oscillating functionals, depending on one or more small-scale parameters that represent the length scales of the heterogeneities.

A common assumption in the literature is based on the premise that the heterogeneities are evenly distributed, leading to the mathematical assumption of periodicity in the so-called fast variable, which encodes the heterogeneities in the mathematical problem. Even though the study of the effective behavior of periodically structured heterogeneous media has enabled the study of more complex ones, it is commonly accepted that periodicity is often not the most well-suited structural hypothesis. This fact is at the basis of many recent works devoted to the study of the effective behavior of random heterogeneous materials whose small-length-scale properties are described

* Received by the editors May 28, 2020; accepted for publication (in revised form) December 9, 2020; published electronically March 25, 2021. Part of this work was carried out while the second and third authors were at the Center for Nonlinear Analysis.

https://doi.org/10.1137/20M1341222

Funding: The work of the second author was partially supported by National Science Foundation grants DMS-1411646 and DMS-1906238. The work of the third author was supported by National Science Foundation grant DMS-1411646, and by an AMS-Simons Travel Award.

${ }^{\dagger}$ King Abdullah University of Science and Technology (KAUST), CEMSE Division, Thuwal 239556900, Saudi Arabia (rita.ferreira@kaust.edu.sa).

‡Department of Mathematical Sciences, Carnegie Mellon University (CMU), Pittsburgh, PA 15213 USA (fonseca@andrew.cmu.edu,rvenkatr@andrew.cmu.edu).

1785 
at a statistical level only.

Here, we are interested in materials with a quasi-crystalline microstructure characterized by small-length-scale properties that are neither periodic nor random. Quasi-crystals, also known as quasi-periodic crystals, are ordered structures that do not share the translational symmetry of traditional crystals [56, 57]. A quasicrystalline pattern can continuously fill an $\mathrm{m}$ - dimensional space but will never be translational symmetric in more than $\mathrm{m}-1$ linearly independent directions.

The discovery of quasi-crystals was announced in the early 1980s by two groups of crystallographers, Shechtman et al. [56] and Levine and Steinhardt [43]. At first, this was received with skepticism, and even hostility, by the scientific community, as quasi-crystals violate the foundations of classical crystallography. However, in 2011, Shechtman was awarded the Nobel Prize in Chemistry for this discovery. A striking feature of quasi-crystals is that their Bragg diffraction displays peculiar five-, ten-, or twelvefold symmetry orders in contrast with the rigid crystallography of periodic crystals. Moreover, the assembly of quasi-crystalline tiling patterns is nonlocal and exhibits similar patterns at different scales (self-similarity).

In various mathematical communities, there has been a rich discussion on quasicrystals, and extensive efforts have been made to model them; see [7, 16, 42, 46] and related references. A well-established mathematical approach for studying quasicrystals is based on aperiodic tilings of hyperplanes, in which one aims at finding a set of geometric shapes, called tiles, paving the Euclidean plane without gaps or overlaps, in a nonperiodic manner only (see Figure 1). A systematic, but not exhaustive, scheme for deriving such tilings is the cut-and-project method, introduced by de Bruijn [26] and further developed by Duneau and Katz [27], which extends Penrose's ideas of aperiodic tilings of the plane [54] to higher dimensions (see [8] for a more detailed description).

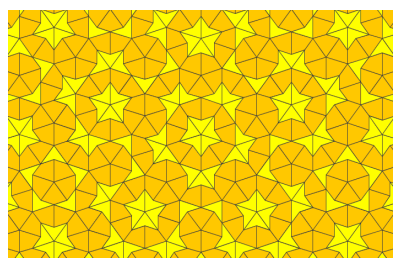

FIG. 1. A quasi-crystalline heterogeneous microstructure corresponding to the so-called "kite and dart" tiling of the plane with fivefold symmetry. Image source: https://rosettacode.org/wiki/ Penrose_tiling.

Roughly speaking, m-dimensional quasi-crystalline patterns can be modeled by cutting periodic tilings in an m-dimensional space, with $\mathrm{m}>\mathrm{m}$, through an $\mathrm{m}$ dimensional subspace with irrational slope. To be precise, given an m-dimensional quasi-crystal $R$ and representing by $\sigma_{R}: \mathbb{R}^{\mathrm{n}} \rightarrow \mathbb{R}$ a constitutive property of $R$, we can find $\mathrm{m} \in \mathbb{N}$, with $\mathrm{m}>\mathrm{m}$, a $Y^{\mathrm{m}}$-periodic function $\sigma: \mathbb{R}^{\mathrm{m}} \rightarrow \mathbb{R}$ with $Y^{\mathrm{m}} \subset \mathbb{R}^{\mathrm{m}}$ a parallelotope, and a linear map $\boldsymbol{R}: \mathbb{R}^{\mathrm{n}} \rightarrow \mathbb{R}^{\mathrm{m}}$ such that

$$
\sigma_{R}(x)=\sigma(\boldsymbol{R} x)
$$

In the homogenization literature, the structural condition (1.1) is referred to as quasiperiodicity $[16,40]$. Here and in what follows, we do not distinguish the linear map from its associated matrix in $\mathbb{R}^{\mathrm{m} \times \mathrm{n}}$, and denote both by $\boldsymbol{R}$. For example (see [35]), 
setting $\tau=\frac{1+\sqrt{5}}{2}$, the matrices

$$
\begin{aligned}
& \boldsymbol{R}=\boldsymbol{R}_{5 m-s y m}:=\frac{1}{\sqrt{10}}\left[\begin{array}{ccc}
\sqrt{5} & 0 & 0 \\
1 & \frac{\sqrt{5}}{\sqrt{2+\tau}} \tau & 1-\tau \\
1 & \frac{\sqrt{5}}{\sqrt{2+\tau}} & \tau \\
1 & -\frac{\sqrt{5}}{\sqrt{2+\tau}} & \tau \\
1 & 0 & -2 \\
-1 & \frac{\sqrt{5}}{\sqrt{2+\tau}} \tau & \tau-1
\end{array}\right] \\
& \boldsymbol{R}=\boldsymbol{R}_{m \overline{3}-s y m}:=\frac{1}{\sqrt{2(\tau+2)}}\left[\begin{array}{ccc}
1 & \tau & 0 \\
\tau & 0 & 1 \\
0 & 1 & \tau \\
-1 & \tau & 0 \\
\tau & 0 & -1 \\
0 & -1 & \tau
\end{array}\right] \\
& \text { and } \boldsymbol{R}=\boldsymbol{R}_{\overline{3} m-s y m}:=\left[\begin{array}{ccc}
\frac{2 \tau+1}{\sqrt{6(4 \tau+3)}} & \frac{-1}{\sqrt{2(\tau+2)}} & \frac{1}{\sqrt{6(\tau+2)}} \\
\frac{2 \tau+1}{\sqrt{6(4 \tau+3)}} & 0 & \frac{-2}{\sqrt{6(\tau+2)}} \\
\frac{2 \tau+1}{\sqrt{6(4 \tau+3)}} & \frac{1}{\sqrt{2(\tau+2)}} & \frac{1}{\sqrt{6(\tau+2)}} \\
\frac{1}{\sqrt{6(4 \tau+3)}} & 0 & \frac{2 \tau}{\sqrt{6(\tau+2)}} \\
\frac{1}{\sqrt{6(4 \tau+3)}} & \frac{-\tau}{\sqrt{2(\tau+2)}} & \frac{-\tau}{\sqrt{6(\tau+2)}} \\
\frac{1}{\sqrt{6(4 \tau+3)}} & \frac{\tau}{\sqrt{2(\tau+2)}} & \frac{-\tau}{\sqrt{6(\tau+2)}}
\end{array}\right]
\end{aligned}
$$

are associated with icosahedral quasi-crystalline patterns exhibiting a pentagonal $\overline{5} m$-symmetry phase, a cubic $m \overline{3}$-symmetry phase, and a rhombohedral trigonal $\overline{3} m$ symmetry phase, respectively, where we adopted the terminology commonly used for the classification of quasi-crystals' symmetry space groups (see, for instance, [59]). These icosahedral quasi-crystalline patterns are found in alloys within, for instance, the Al-Mn-Si, the Al-Li-Cu, and the Al-Cu-Fe ternary systems (see [35]).

In general, there are multiple choices for $\mathrm{m}, \sigma$, and $\boldsymbol{R}$, which could lead to some ambiguity in our asymptotic analysis. However, as proved in [8], the homogenization analysis does not depend on $\boldsymbol{R}$ provided it satisfies the diophantine condition

$$
\boldsymbol{R}^{*} k \neq 0 \text { for all } k \in \mathbb{Z}^{\mathrm{m}} \backslash\{0\},
$$

where $\boldsymbol{R}^{*}$ denotes the transpose of $\boldsymbol{R}$. This condition implies that some entries of $\boldsymbol{R}$ must be irrational, which justifies the expression irrational slope used above.

Quasi-crystalline composites and alloys have played a central role in materials science and other areas of engineering [4, 6, 28, 36, 38, 39, 49, 61]. Indeed, Al-Cu-Fe quasi-crystalline materials in polymer-based composites have been shown to significantly improve wear-resistance to volume loss, and a twofold increase in the elastic moduli. As we mentioned before, the mathematical study of such quasi-crystalline composites does not fit within the classical periodic homogenization theory. More appropriate in the context of quasi-crystal composites are almost-periodic and stochastic homogenization, which were initiated with the works of Papanicolaou and Varadhan [53], Kozlov [40], and Oleı̆nik and Zhikov [52] for partial differential equations, Modica and Dal Maso [47] and Braides [9, 10, 11] within a variational framework; see also $[12,17,25]$ and the references therein. However, such approaches do 
not take full advantage of the quasi-crystalline feature of the problem, often leading to asymptotic formulas that pose computational difficulties and are not stable under perturbations. Instead, we adopt and further develop a homogenization procedure based on the two-scale-cut-and-project convergence introduced in [8], and recently revisited in [60], which leads to a more tractable (even though higher-dimensional) cell problem.

In this paper, we initiate a research program that is devoted to the study of quasi-crystalline homogenization problems involving oscillating integral energies under quasi-crystalline oscillating differential constraints and falls within the framework of $\mathcal{A}$-quasiconvexity. To be precise, we aim at characterizing the asymptotic behavior of integral energies of the form

$$
F_{\varepsilon}\left(u_{\varepsilon}\right):=\int_{\Omega} f_{R}\left(x, \frac{x}{\varepsilon^{\alpha}}, u_{\varepsilon}(x)\right) \mathrm{d} x
$$

as $\varepsilon \rightarrow 0^{+}$, where $\varepsilon^{\alpha}>0$, with $\alpha \geqslant 0$, represents the length-scale of the tiles featuring the quasi-crystalline composite. Moreover, $\Omega \subset \mathbb{R}^{\mathrm{n}}$, with $\mathrm{m} \in \mathbb{N}$, is an open and bounded set that represents the container occupied by the composite, and $f_{R}$ is the Lagrangian of the system whose dependence on the second variable, the fast variable, encodes the quasi-crystalline structure of the composite, indicated with the subscript $R$ as in (1.1). Finally, $u_{\varepsilon}$ is an abstract vector-valued order-parameter whose physical interpretation might depend on the problem in question. A typical case is that in which $u_{\varepsilon}$ is curl-free, and $u_{\varepsilon}=\nabla v_{\varepsilon}$ for some potential deformation $v_{\varepsilon}$. However, many applications require that $u_{\varepsilon}$ instead satisfies other linear partial differential constraints, such as Maxwell's equations in the case of electromagnetism, or, in the case of linear elasticity, that $u_{\varepsilon}$ be the symmetric part of a gradient. A unified abstract approach to several of these constraints is that of $\mathcal{A}$-free fields, as pioneered by Fonseca and Müller [32] (see also $[20,21,55]$ ). To be precise, $u_{\varepsilon} \in L^{p}\left(\Omega ; \mathbb{R}^{\mathbb{d}}\right)$ is subject to quasi-crystalline oscillating differential constraints such as

$$
\mathcal{A}_{\varepsilon} u_{\varepsilon}:=\sum_{i=1}^{\mathrm{m}} A_{R}^{i}\left(\frac{\cdot}{\varepsilon^{\beta}}\right) \frac{\partial u_{\varepsilon}}{\partial x_{i}}(\cdot) \rightarrow 0 \text { strongly in } W^{-1, p}\left(\Omega ; \mathbb{R}^{\mathbb{1}}\right)
$$

or, in divergence form,

$$
\mathcal{A}_{\varepsilon} u_{\varepsilon}:=\sum_{i=1}^{\mathrm{n}} \frac{\partial}{\partial x_{i}}\left(A_{R}^{i}\left(\frac{\dot{ }}{\varepsilon^{\beta}}\right) u_{\varepsilon}(\cdot)\right) \rightarrow 0 \quad \text { strongly in } W^{-1, p}\left(\Omega ; \mathbb{R}^{\mathbb{1}}\right),
$$

with $\mathbb{d}, \mathbb{1} \in \mathbb{N}$ and $1<p<\infty$, where for every $x \in \mathbb{R}^{\mathrm{n}}, A_{R}^{i}(x) \in \operatorname{Lin}\left(\mathbb{R}^{\mathbb{d}} ; \mathbb{R}^{\mathbb{1}}\right)$ features a quasi-crystalline pattern, and $\beta \geqslant 0$ is a parameter. For the study of homogenization of integral energies with periodic energy densities and under periodically oscillating $\mathcal{A}$-free differential constraints, we refer the reader to [13, 23, 24, 31, 41, 45].

As in the periodic setting $[23,24]$, we expect different asymptotic regimes according to the ratio between $\alpha$ and $\beta$. As a starting point to this extensive research project, we first focus here on the case where $\beta=0$ and $A_{R}^{i}$ is independent of $x$, in which case $u_{\varepsilon}$ is subjected to homogeneous first-order linear partial differential constraints. Precisely, in this paper we address the problem of characterizing the asymptotic behavior as $\varepsilon \rightarrow 0^{+}$of integral energies of the form

$$
F_{\varepsilon}(u):=\int_{\Omega} f_{R}\left(x, \frac{x}{\varepsilon}, u(x)\right) \mathrm{d} x
$$

Copyright $@$ by SIAM. Unauthorized reproduction of this article is prohibited. 
for $u \in L^{p}\left(\Omega ; \mathbb{R}^{\mathbb{d}}\right)$ satisfying $\mathcal{A} u=0$, where

$$
\mathcal{A} u:=\sum_{i=1}^{\mathrm{m}} A^{(i)} \frac{\partial u}{\partial x_{i}} \quad \text { with } A^{(i)} \in \mathbb{R}^{\mathbb{1} \times \mathbb{d}} \text { for all } i \in\{1, \ldots, \mathrm{m}\} .
$$

We refer the reader to section 2.2 for a rigorous definition of the identity $\mathcal{A} u=0$, in which case we say that the vector field $u$ is $\mathcal{A}$-free (see Definition 2.1). A common assumption within studies involving $\mathcal{A}$-free vector fields is the constant-rank property, which states that there exists $r \in \mathbb{N}$ such that for all $w \in \mathbb{R}^{\mathrm{n}} \backslash\{0\}$, we have

$$
\operatorname{rank} \mathbb{A}(w)=r,
$$

where $\mathbb{A}: \mathbb{R}^{\mathrm{n}} \rightarrow \mathbb{R}^{\mathbb{1} \times \mathbb{d}}$ denotes the symbol of $\mathcal{A}$ and is defined by

$$
\mathrm{A}(w):=\sum_{i=1}^{\mathrm{m}} A^{(i)} w_{i}
$$

for $w \in \mathbb{R}^{\mathrm{m}}$. We assume that our operator $\mathcal{A}$ satisfies the constant-rank property, and we refer the reader to $[32,48,58]$ for further insights on this property and on $\mathcal{A}$-free fields.

Our asymptotic analysis of the energy integrals in (1.4) under the constraint (1.5) is based on $\Gamma$-convergence techniques, whose key point is to find an integral representation for

$$
F_{\text {hom }}(u):=\inf \left\{\liminf _{\varepsilon \rightarrow 0^{+}} F_{\varepsilon}\left(u_{\varepsilon}\right): u_{\varepsilon} \rightarrow u \text { in } L^{p}\left(\Omega ; \mathbb{R}^{\mathrm{d}}\right), \mathcal{A} u_{\varepsilon}=0\right\} .
$$

To state our main theorem regarding this integral representation, we first introduce the hypotheses on the Lagrangian, $f_{R}: \Omega \times \mathbb{R}^{\mathrm{m}} \times \mathbb{R}^{\mathrm{d}} \rightarrow[0, \infty)$ :

(H1) (quasi-crystallinity): There exist $\mathrm{m} \in \mathbb{N}$, with $\mathrm{m}>\mathrm{m}$, a matrix $\boldsymbol{R} \in \mathbb{R}^{\mathrm{m} \times \mathrm{n}}$ satisfying (1.2), and a continuous function $f: \Omega \times \mathbb{R}^{\mathrm{m}} \times \mathbb{R}^{\mathrm{d}} \rightarrow[0, \infty)$ such that the function $f(x, \cdot, \xi)$ is $Y^{\mathrm{m}}$-periodic for each $(x, \xi) \in \Omega \times \mathbb{R}^{\mathrm{d}}$, with $Y^{\mathrm{m}}$ denoting a parallelotope in $\mathbb{R}^{\mathrm{m}}$, and

$$
f_{R}(x, z, \xi)=f(x, \boldsymbol{R} z, \xi)
$$

for all $(x, z, \xi) \in \Omega \times \mathbb{R}^{\mathrm{n}} \times \mathbb{R}^{\mathrm{d}}$.

(H2) (growth): There exist $p \in(1, \infty)$ and $C>0$ such that

$$
0 \leqslant f_{R}(x, z, \xi) \leqslant C\left(1+|\xi|^{p}\right)
$$

for all $(x, z, \xi) \in \Omega \times \mathbb{R}^{\mathrm{n}} \times \mathbb{R}^{\mathrm{d}}$.

In the proof of the lower bound for the integral representation of $F_{\text {hom }}$, we will require an additional hypothesis:

(H3) (convexity): For all $(x, y) \in \Omega \times \mathbb{R}^{\mathrm{m}}$, the function $\xi \mapsto f(x, y, \xi)$ is convex and $C^{1}$.

We refer the reader to section 2 for a list of the main notation we use in this paper. However, for the readability of our main results, we clarify upfront that $L_{\#}^{p}\left(Y^{\mathrm{m}} ; \mathbb{R}^{\mathrm{m}}\right)$ denotes the space of $Y^{\mathrm{m}}$-periodic functions belonging to $L_{\mathrm{loc}}^{p}\left(\mathbb{R}^{\mathrm{m}}\right)$. Moreover, given a Lebesgue measurable set $B \subset \mathbb{R}^{\mathrm{k}}$, with $\mathbb{k} \in \mathbb{N}$, we use the notation $f_{B}$. in place of $\frac{1}{\mathcal{L}^{\mathrm{k}}(B)} \int_{B} \cdot$, where $\mathcal{L}^{\mathrm{k}}(B)$ denotes the $\mathbb{k}$-dimensional Lebesgue measure of $B$. 
TheOREM 1.1. Let $\Omega \subset \mathbb{R}^{\mathrm{n}}$ be an open and bounded set, let $f_{R}: \Omega \times \mathbb{R}^{\mathrm{n}} \times \mathbb{R}^{\mathrm{d}} \rightarrow$ $[0, \infty)$ be a function satisfying $(\mathrm{H} 1)-(\mathrm{H} 3)$, let $F_{\mathrm{hom}}$ be the functional introduced in (1.8), and assume that (1.6) holds. Then, for all

$$
u \in \mathcal{U}_{\mathcal{A}}:=\left\{u \in L^{p}\left(\Omega ; \mathbb{R}^{\mathrm{d}}\right): \mathcal{A} u=0\right\},
$$

we have

$$
F_{\text {hom }}(u)=\int_{\Omega} f_{\text {hom }}(x, u(x)) \mathrm{d} x
$$

where

$$
f_{\text {hom }}(x, \xi):=\inf _{v \in \mathcal{V}_{\mathcal{A}}} f_{Y^{\mathrm{m}}} f(x, y, \xi+v(y)) \mathrm{d} y,
$$

with

(1.10)

$$
\begin{aligned}
& \mathcal{V}_{\mathcal{A}}:=\left\{v \in L_{\#}^{p}\left(Y^{\mathrm{m}} ; \mathbb{R}^{\mathrm{d}}\right): v \text { is } \mathcal{A}_{\boldsymbol{R}^{*} \text {-free in the sense of Definition } 3.11 \text { and }}\right. \\
& \left.\qquad \int_{Y^{\mathrm{m}}} v(y) \mathrm{d} y=0\right\} .
\end{aligned}
$$

Remark 1.2 (on the hypotheses of Theorem 1.1). (i) In the homogenization literature, measurability of $f$ with respect to the fast variable is often preferred over continuity. As we further discuss in section 2.1, measurability of $f_{R}$ requires, in general, Borel-measurability of $f$. A common approach for dealing with lack of continuity is to combine periodicity with Scorza-Dragoni type results that, up to a set of small measure, allow us to reduce the problem to the continuity setting. Here, however, we cannot use such an argument because a set of small m-dimensional Lebesgue measure, the ambient space for the fast variable in terms of (the periodic function) $f$, may not have small $\mathrm{m}$-dimensional Lebesgue, the ambient space for the fast variable in terms of (the quasi-crystalline function) $f_{R}$. (ii) The nonconvex case raises nontrivial difficulties in the quasi-crystalline setting and will be the subject of a forthcoming work.

In the Sobolev setting, homogenization of integral energies of the form (1.4) under nonperiodic assumptions was undertaken in $[12,37,47]$ in the $\mathcal{A}=$ curl case, assuming coercivity. Within the quasi-crystalline framework, Theorem 1.1 extends these results to the general $\mathcal{A}$-free setting and without coercivity. We prove Theorem 1.1 in section 4; the main tools we use here are based on $\Gamma$-convergence and on two-scale convergence adapted to the quasi-crystalline setting, also called two-scale-cut-andproject convergence. For brevity, and having in mind the relation (1.1), we refer to the two-scale-cut-and-project convergence as $\boldsymbol{R}$-two-scale convergence. This notion was introduced in [8] (also see [60]) as an extension of the usual notion of two-scale convergence $[1,51]$ to enable the study of composites whose underlying microstructure has a quasi-crystalline feature.

Here, we further extend the study of $\boldsymbol{R}$-two-scale convergence in two different ways. In $[8,60]$, the authors consider sequences in $L^{2}$, and their arguments are based on Fourier analysis relying heavily on Parseval's and Plancherel's identities. Instead, we consider the more general case of $L^{p}$ with $p \in(1, \infty)$. Moreover, in [8] the authors characterize the limit, with respect to the $\boldsymbol{R}$-two-scale convergence, of 
bounded sequences in $W^{1,2}$, while in [60] the authors characterize the limit associated with bounded sequences in $L^{2}$ that are divergence-free or curl-free. Here, besides generalizing these results to the $L^{p}$ case, we provide a unified approach to all these cases by considering bounded sequences in $L^{p}$ that are $\mathcal{A}$-free, in the spirit of [31] concerning the periodic case.

Next, we state our main result regarding the characterization of the limits of bounded sequences in $L^{p}$ that are $\mathcal{A}$-free. We refer the reader to sections 2.2 and 3.1, where we give precise meanings of the expressions " $\mathcal{A}$-free" and " $\left(\mathcal{A}, \mathcal{A}_{\boldsymbol{R}^{*}}^{y}\right)$-free" that we make use of in the following statement.

TheOREM 1.3. Let $\boldsymbol{R} \in \mathbb{R}^{\mathrm{m} \times \mathrm{m}}$ satisfy (1.2). A function $u \in L^{p}\left(\Omega \times Y^{\mathrm{m}} ; \mathbb{R}^{\mathrm{d}}\right)$ is the $\boldsymbol{R}$-two-scale limit of an $\mathcal{A}$-free sequence $\left\{u_{\varepsilon}\right\}_{\varepsilon} \subset L^{p}\left(\Omega ; \mathbb{R}^{\mathbb{d}}\right)$ if and only if $u$ is $\left(\mathcal{A}, \mathcal{A}_{\boldsymbol{R}^{*}}^{y}\right)$-free in the sense of Definition 3.13 ; that is,

$$
\mathcal{A} \bar{u}_{0}=0 \quad \text { and } \quad \mathcal{A}_{\boldsymbol{R}^{*}}^{y} \bar{u}_{1}=0
$$

in the sense of Definitions 2.1 and 3.11 , respectively, where $\bar{u}_{0}:=f_{Y^{\mathrm{m}}} u(\cdot, y) \mathrm{d} y$ and $\bar{u}_{1}:=u-\bar{u}_{0}$.

We prove Theorem 1.3 in section 3.1, where we use arguments similar to those in [31] concerning the periodic case (see [31, Theorem 2.12]). We observe that the sufficient part in Theorem 1.3, which guarantees that (1.11) fully characterizes the $\boldsymbol{R}$-two-scale limits, is new in the literature even for $p=2$ and $\mathcal{A}=$ curl or $\mathcal{A}=$ div treated in $[8,60]$. Furthermore, in section 5 we give an alternative proof of Theorem 1.3 for the $\mathcal{A}=$ curl case using arguments based on Fourier analysis that differ from those in $[8,60]$ because Parseval's and Plancherel's identities do not hold for $p \neq 2$. This alternative proof provides an equivalent alternative characterization for the $\boldsymbol{R}$-two-scale limit of bounded sequences in $W^{1, p}$ and may provide useful arguments for studying homogenization problems involving quasi-crystalline functionals in the $\mathcal{A}=$ curl case. This alternative characterization can be stated as follows.

TheOREm 1.4. Let $\boldsymbol{R} \in \mathbb{R}^{\mathrm{m} \times \mathrm{m}}$ satisfy (1.2), and let $Y^{\mathrm{m}} \subset \mathbb{R}^{\mathrm{m}}$ be a parallelotope. Then, a function $v \in L^{p}\left(\Omega \times Y^{\mathrm{m}} ; \mathbb{R}^{\mathrm{m}}\right)$ is the $\boldsymbol{R}$-two-scale limit of a sequence $\left\{\nabla v_{\varepsilon}\right\}_{\varepsilon}$ with $\left\{v_{\varepsilon}\right\}_{\varepsilon}$ bounded in $W^{1, p}(\Omega)$ if and only if there exist $v_{0} \in W^{1, p}(\Omega)$ and $v_{1} \in$ $L^{p}\left(\Omega ; \mathcal{G}_{\boldsymbol{R}}^{p}\right)$ such that

$$
v=\nabla v_{0}+v_{1}
$$

where

$$
\mathcal{G}_{\boldsymbol{R}}^{p}:=\left\{w \in L_{\#}^{p}\left(Y^{\mathrm{m}} ; \mathbb{R}^{\mathrm{m}}\right): \hat{w}_{k}=\lambda_{k} \boldsymbol{R}^{*} k \text { for some }\left\{\lambda_{k}\right\}_{k \in \mathbb{Z}^{\mathrm{m}}} \subset \mathbb{C} \text { with } \lambda_{0}=0\right\},
$$

with $\hat{w}_{k}:=f_{Y^{\mathrm{m}}} w(y) e^{-2 \pi i k \cdot y} \mathrm{~d} y, k \in \mathbb{Z}^{\mathrm{m}}$, denoting the Fourier coefficients of $w$.

Remark 1.5. We recall that if $u_{\varepsilon} \in L^{p}\left(\Omega ; \mathbb{R}^{\mathrm{n}}\right)$ is curl-free in $\mathbb{R}^{\mathrm{n}}$ with $\Omega$ simply connected, then there exists $v_{\varepsilon} \in W^{1, p}(\Omega)$ such that $u_{\varepsilon}=\nabla v_{\varepsilon}$. Thus, in terms of the notation in the two previous results with $\mathbb{d}=\mathrm{n}$, we have $\bar{u}_{0}=\nabla v_{0}$ and $\bar{u}_{1}=v_{1}$. In particular, (1.12) provides an alternative characterization of $\mathcal{A}_{\boldsymbol{R}^{*-}}$ and $\mathcal{A}_{\boldsymbol{R}^{*}}^{y}$-free vector fields introduced in Definition 3.11 in the $\mathcal{A}=$ curl case (also see Remark 5.7 for a detailed argumentation).

2. Notation and preliminaries. Throughout this paper, $m, m \in \mathbb{N}$ are such that $\mathrm{m}>\mathrm{m}, \Omega \subset \mathbb{R}^{\mathrm{m}}$ is an open and bounded set, $Y^{\mathrm{m}}$ is a parallelotope in $\mathbb{R}^{\mathrm{m}}$, 
$\Pi \subset \mathbb{R}^{\mathrm{n}}$ is a parallelotope in $\mathbb{R}^{\mathrm{n}}, \mathbb{d}, \mathbb{1} \in \mathbb{N}$, and $p, p^{\prime} \in(1, \infty)$ are such that $\frac{1}{p}+\frac{1}{p^{\prime}}=1$. Moreover, we assume that $\varepsilon$ takes values on an arbitrary sequence of positive numbers that converges to zero.

We use the subscript \# within function spaces to highlight an underlying periodicity, in which case the domain indicates the periodicity cell. For instance, $C_{\#}\left(Y^{\mathrm{m}}\right)=$ $\left\{u \in C\left(\mathbb{R}^{\mathrm{m}}\right): u\right.$ is $Y^{\mathrm{m}}$-periodic $\}$ and $L_{\#}^{p}(\Pi)=\left\{u \in L_{\mathrm{loc}}^{p}\left(\mathbb{R}^{\mathrm{n}}\right): u\right.$ is $\Pi$-periodic $\}$.

Next, we compile the notation and main properties of the cut-and-project maps $\boldsymbol{R}$ and differential operators $\mathcal{A}$ presented in the introduction and that we make use of in what follows.

2.1. Cut-and-project maps $\boldsymbol{R}$. In this paper, $\boldsymbol{R}: \mathbb{R}^{\mathrm{n}} \rightarrow \mathbb{R}^{\mathrm{m}}$ is a linear map, whose associated matrix in $\mathbb{R}^{\mathrm{m} \times \mathrm{m}}$ is also denoted by $\boldsymbol{R}$. We do not distinguish between the transpose matrix and the adjoint of $\boldsymbol{R}$, and denote both by $\boldsymbol{R}^{*}$. We often assume that the criterion (1.2) on $\boldsymbol{R}$ holds, in which case we refer to it explicitly.

As shown in [8], if $g: \mathbb{R}^{\mathrm{m}} \rightarrow \mathbb{R}$ is a $Y^{\mathrm{m}}$-periodic trigonometric polynomial, with $Y^{\mathrm{m}}$ a parallelotope in $\mathbb{R}^{\mathrm{m}}$, then the ergodic mean of $g \circ \boldsymbol{R}, \mathcal{M}(g \circ \boldsymbol{R})$, is uniquely defined provided that $\boldsymbol{R}$ satisfies (1.2), in which case we have

$$
\mathcal{M}(g \circ \boldsymbol{R}):=\lim _{\tau \rightarrow \infty} \frac{1}{(2 \tau)^{\mathrm{n}}} \int_{(-\tau, \tau)^{\mathrm{n}}} g(\boldsymbol{R} x) \mathrm{d} x=f_{Y^{\mathrm{m}}} g(y) \mathrm{d} y .
$$

Throughout this paper, we consider functions $\sigma_{R}$ as in (1.1). We observe that such a definition raises measurability issues. In fact, we can only guarantee that $\sigma_{R}$ in (1.1) is measurable provided that $\sigma$ is Borel-measurable. We conjecture that there are functions $\sigma \in L^{\infty}\left(\mathbb{R}^{\mathrm{m}}\right)$ for which the corresponding function $\sigma_{R}$ in (1.1) is not measurable. This conjecture is based upon the observation that the preimage of a measurable set $B \subset \mathbb{R}^{\mathrm{m}}$ through $R, R^{-1}(B)$, acts as a projection of the set $B$ onto the lower-dimensional space $\mathbb{R}^{\mathrm{n}}$; moreover, as is well known, the projection of a measurable set may not be measurable. To overcome this issue, we take in (1.1) the Borel representative of $\sigma$.

2.2. Differential operators $\mathcal{A}$ with constant coefficients. We consider homogeneous first-order linear partial differential operators with constant coefficients, $\mathcal{A}$, that map $u: \Omega \rightarrow \mathbb{R}^{\mathbb{d}}$ into $\mathcal{A} u: \Omega \rightarrow \mathbb{R}^{\mathbb{1}}$, of the form

$$
\mathcal{A} u:=\sum_{i=1}^{\mathrm{n}} A^{(i)} \frac{\partial u}{\partial x_{i}} \quad \text { with } A^{(i)} \in \mathbb{R}^{\mathbb{1} \times \mathbb{d}} \text { for all } i \in\{1, \ldots, \mathbb{n}\} .
$$

The formal adjoint of $\mathcal{A}$, which we denote by $\mathcal{A}^{*}$, maps $v: \Omega \rightarrow \mathbb{R}^{\mathbb{1}}$ into $\mathcal{A}^{*} v: \Omega \rightarrow \mathbb{R}^{\mathrm{d}}$ and is defined by

$$
\mathcal{A}^{*} v:=-\sum_{i=1}^{\mathrm{n}}\left(A^{(i)}\right)^{T} \frac{\partial v}{\partial x_{i}} .
$$

We observe that $\mathcal{A}$ can be viewed as a bounded, linear operator $\mathcal{A}: L^{p}\left(\Omega ; \mathbb{R}^{\mathrm{d}}\right) \rightarrow$ $W^{-1, p}\left(\Omega ; \mathbb{R}^{\mathbb{1}}\right)$ by setting

$$
\langle\mathcal{A} u, v\rangle:=\int_{\Omega} u \cdot \mathcal{A}^{*} v \mathrm{~d} x
$$

for all $u \in L^{p}\left(\Omega ; \mathbb{R}^{\mathbb{d}}\right)$ and $v \in W_{0}^{1, p^{\prime}}\left(\Omega ; \mathbb{R}^{\mathbb{1}}\right)$. We observe further that if $u \in C_{c}^{1}\left(\Omega ; \mathbb{R}^{\mathbb{d}}\right)$ 
and $v \in C_{c}^{1}\left(\Omega ; \mathbb{R}^{\mathbb{1}}\right)$, then

$$
\int_{\Omega} \mathcal{A} u \cdot v \mathrm{~d} x=\int_{\Omega} u \cdot \mathcal{A}^{*} v \mathrm{~d} x
$$

by integration by parts. Similarly, if $u \in C_{\#}^{1}\left(\Pi ; \mathbb{R}^{\mathbb{d}}\right)$ and $v \in C_{\#}^{1}\left(\Pi ; \mathbb{R}^{\mathbb{1}}\right)$, then

$$
\int_{\Pi} \mathcal{A} u \cdot v \mathrm{~d} x=\int_{\Pi} u \cdot \mathcal{A}^{*} v \mathrm{~d} x .
$$

We assume that $\mathcal{A}$ satisfies the constant-rank property, that is, there exists $r \in \mathbb{N}$ such that for all $w \in \mathbb{R}^{\mathrm{n}} \backslash\{0\}$, we have rank $\mathbb{A}(w)=r$, where $\mathbb{A}: \mathbb{R}^{\mathrm{n}} \rightarrow \mathbb{R}^{\mathbb{1} \times \mathbb{d}}$ denotes the symbol of $\mathcal{A}$, and is defined by (1.7). As we mentioned in the introduction, the constant-rank property is a common assumption within studies involving $\mathcal{A}$-free vector fields. We refer the reader to $[32,48,58]$ for further insights on this property and on $\mathcal{A}$-free fields, whose notion we recall next.

Definition 2.1 ( $\mathcal{A}$-free fields). (i) Given $u \in L^{p}\left(\Omega ; \mathbb{R}^{\mathbb{d}}\right)$, we say that $\mathcal{A} u$ exists in $L^{p}\left(\Omega ; \mathbb{R}^{\mathbb{1}}\right)$ if there exists a function $U \in L^{p}\left(\Omega ; \mathbb{R}^{\mathbb{1}}\right)$ such that, for every $\phi \in C_{c}^{1}\left(\Omega ; \mathbb{R}^{\mathbb{1}}\right)$, we have

$$
\int_{\Omega} u \cdot \mathcal{A}^{*} \phi \mathrm{d} x=\int_{\Omega} U \cdot \phi \mathrm{d} x
$$

In this case, we write $\mathcal{A} u:=U$. We say that $u$ is $\mathcal{A}$-free, and write $\mathcal{A} u=0$, if (2.1) holds with $U=0$.

(ii) Given $v \in L_{\#}^{p}\left(\Pi ; \mathbb{R}^{\mathrm{d}}\right)$, we say that $\mathcal{A}$ v exists in $L_{\#}^{p}\left(\Pi ; \mathbb{R}^{\mathbb{1}}\right)$ if there exists a function $V \in L_{\#}^{p}\left(\Pi ; \mathbb{R}^{\mathbb{1}}\right)$ such that, for every $\varphi \in C_{\#}^{1}\left(\Pi ; \mathbb{R}^{\mathbb{1}}\right)$, we have

$$
\int_{\Pi} u \cdot \mathcal{A}^{*} \varphi \mathrm{d} y=\int_{\Pi} V \cdot \varphi \mathrm{d} y .
$$

In this case, we write $\mathcal{A} v:=V$. We say that $v$ is $\mathcal{A}$-free, and write $\mathcal{A} v=0$, if (2.2) holds with $V=0$.

Remark 2.2 ( $\mathcal{A}$ applied to vector fields depending on several variables). Whenever a vector field depends on two or more variables, we index $\mathcal{A}$ with the underlying variable to which $\mathcal{A}$ is being applied to the vector field. For instance, if $u=u(x, y)$, then $\mathcal{A}_{x} u$ refers to $\mathcal{A}$ applied to $u$ as a function of $x$ with $y$ regarded as a fixed parameter. Similarly, $\mathcal{A}_{y} u$ refers to $\mathcal{A}$ applied to $u$ as a function of $y$ with $x$ regarded as a fixed parameter.

A crucial result in the variational theory of $\mathcal{A}$-free fields is the following $\mathcal{A}$-free periodic extension lemma, established in [32, Lemma 2.15]. We make repeated use of a similar statement, also proved in [31, Lemma 2.8], and hence record it here for the reader's convenience.

Lemma 2.3 ( $\mathcal{A}$-free periodic extension). Let $\Pi \subset \mathbb{R}^{\mathrm{n}}$ be a parallelotope, let $O \subset \Pi$ be an open set, let $1<p<\infty$, and assume that $\mathcal{A}$ satisfies (1.6). Let $\left\{v_{n}\right\} \subset L^{p}\left(O ; \mathbb{R}^{\mathrm{d}}\right)$ be a p-equi-integrable sequence in $O$, with $v_{n} \rightarrow 0$ in $L^{p}\left(O ; \mathbb{R}^{\mathrm{d}}\right)$ and $\mathcal{A} v_{n} \rightarrow 0$ in $W^{-1, p}\left(O ; \mathbb{R}^{\mathbb{1}}\right)$. Then, there exist an $\mathcal{A}$-free sequence $\left\{u_{n}\right\} \subset L_{\#}^{p}\left(\Pi ; \mathbb{R}^{\mathrm{d}}\right)$, which is p-equi-integrable in $\Pi$, and a positive constant $C=C(\mathcal{A})$ such that

$$
\begin{aligned}
& u_{n}-v_{n} \rightarrow 0 \text { in } L^{p}\left(O ; \mathbb{R}^{\mathrm{d}}\right), \quad u_{n} \rightarrow 0 \text { in } L^{p}\left(\Pi \backslash O ; \mathbb{R}^{\mathrm{d}}\right), \quad f_{\Pi} u_{n} \mathrm{~d} y=0, \\
& \left\|u_{n}\right\|_{L^{p}\left(\Pi ; \mathbb{R}^{\mathrm{d}}\right)} \leqslant C\left\|v_{n}\right\|_{L^{p}\left(O ; \mathbb{R}^{\mathrm{d}}\right)} \text { for all } n \in \mathbb{N} .
\end{aligned}
$$

Copyright $\odot$ by SIAM. Unauthorized reproduction of this article is prohibited. 
Proof. The proof of this lemma with $\Pi=(0,1)^{\mathrm{m}}$ can be found in [32, Lemma 2.15] and [31, Lemma 2.8]. The case in which $\Pi$ is an arbitrary parallelotope follows by an affine change of variables.

3. Cut-and-project-two-scale convergence. The notion of two-scale convergence was first introduced in the $L^{2}$ setting by Nguetseng [51] and further developed by Allaire [1]. Initially, it was used to provide a mathematically rigorous justification of the formal asymptotic expansions that are commonly adopted in the study of homogenization problems. Posteriorly, the notion of two-scale convergence was extended, in particular, to $L^{p}, L^{1}, B V$, and Besicovitch spaces [3, 15, 17,30,44], and also to the multiple-scales case $[2,29,33]$ that enhanced several variational homogenization studies hinging on a $\Gamma$-convergence approach, such as $[5,19,30,33,50]$.

In this section, we first address the study of the notion of two-scale convergence in the quasi-crystalline setting, which we refer to as cut-and-project-two-scale convergence (or, for brevity, $\boldsymbol{R}$-two-scale convergence), with $\boldsymbol{R}$ as in section 2.1. We then prove Theorem 1.3.

As we mentioned in the introduction, the $\boldsymbol{R}$-two-scale convergence was introduced in [8] (also see [60]) as an extension of the usual notion of two-scale convergence to enable the study of composites whose underlying microstructure has a quasi-crystalline feature. Using arguments based on Fourier analysis, the authors in [8] characterize the limit, with respect to the $\boldsymbol{R}$-two-scale convergence, of bounded sequences in $W^{1,2}$, while the authors in [60] characterize the limit associated with bounded sequences in $L^{2}$ that are divergence-free or curl-free. Here, besides generalizing these results to the $L^{p}$ setting, with $1<p<\infty$, we provide a unified approach to all these cases by considering bounded sequences in $L^{p}$ that are $\mathcal{A}$-free, with $\mathcal{A}$ as in section 2.2. Our arguments are close to those in [31] concerning the periodic case and hinge on properties of $\mathcal{A}$-free vector fields.

We first introduce the definition of $\boldsymbol{R}$-two-scale convergence in $L^{p}\left(\Omega ; \mathbb{R}^{\mathbb{k}}\right)$. We make use of the results in this section with $\mathbb{k}$ equal to either $1, \mathbb{d}, \mathbb{1}$, or $\mathrm{m}$.

Definition 3.1 ( $\boldsymbol{R}$-two-scale convergence). We say that a sequence $\left\{u_{\varepsilon}\right\}_{\varepsilon} \subset$ $L^{p}\left(\Omega ; \mathbb{R}^{\mathrm{k}}\right) \boldsymbol{R}$-two-scale converges to a function $u \in L^{p}\left(\Omega \times Y^{\mathrm{m}} ; \mathbb{R}^{\mathrm{k}}\right)$ if for all $\varphi \in$ $L^{p^{\prime}}\left(\Omega ; C_{\#}\left(Y^{\mathrm{m}} ; \mathbb{R}^{\mathrm{k}}\right)\right)$ we have

$$
\lim _{\varepsilon \rightarrow 0^{+}} \int_{\Omega} u_{\varepsilon}(x) \cdot \varphi\left(x, \frac{\boldsymbol{R} x}{\varepsilon}\right) \mathrm{d} x=\int_{\Omega} f_{Y^{\mathrm{m}}} u(x, y) \cdot \varphi(x, y) \mathrm{d} x \mathrm{~d} y,
$$

and we write $u_{\varepsilon} \stackrel{\boldsymbol{R}-2 s c}{\longrightarrow} u$.

Remark 3.2 (uniqueness of $\boldsymbol{R}$-two-scale limits). There is uniqueness of the $\boldsymbol{R}$ two-scale limit. In fact, if $\left\{u_{\varepsilon}\right\}_{\varepsilon} \subset L^{p}\left(\Omega ; \mathbb{R}^{\mathrm{k}}\right)$ and $u, \tilde{u} \in L^{p}\left(\Omega \times Y^{\mathrm{m}} ; \mathbb{R}^{\mathrm{k}}\right)$ are such that $u_{\varepsilon} \stackrel{\boldsymbol{R}-2 s c}{\longrightarrow} u$ and $u_{\varepsilon} \stackrel{R-2 s c}{\longrightarrow} \tilde{u}$, then

$$
\int_{\Omega} f_{Y^{\mathrm{m}}}(u(x, y)-\tilde{u}(x, y)) \cdot \varphi(x, y) \mathrm{d} x \mathrm{~d} y=0
$$

for all $\varphi \in L^{p^{\prime}}\left(\Omega ; C_{\#}\left(Y^{\mathrm{m}} ; \mathbb{R}^{\mathrm{k}}\right)\right)$. Hence, $u=\tilde{u}$ a.e. in $\Omega \times Y^{\mathrm{m}}$.

Remark 3.3 (on the test functions for $\boldsymbol{R}$-two-scale convergence). Assume that $\left\{u_{\varepsilon}\right\}_{\varepsilon}$ is a bounded sequence in $L^{p}\left(\Omega ; \mathbb{R}^{\mathbb{k}}\right)$. Then, $\left\{u_{\varepsilon}\right\}_{\varepsilon} \boldsymbol{R}$-two-scale converges to a function $u \in L^{p}\left(\Omega \times Y^{\mathrm{m}} ; \mathbb{R}^{\mathrm{k}}\right)$ if and only if (3.1) holds for all $\varphi \in C_{c}^{\infty}\left(\Omega ; C_{\#}^{\infty}\left(Y^{\mathrm{m}} ; \mathbb{R}^{\mathrm{k}}\right)\right)$. To prove this statement, it suffices to use the density of $C_{c}^{\infty}\left(\Omega ; C_{\#}^{\infty}\left(Y^{\mathrm{m}} ; \mathbb{R}^{\mathrm{k}}\right)\right)$ in $L^{p^{\prime}}\left(\Omega ; C_{\#}\left(Y^{\mathrm{m}} ; \mathbb{R}^{\mathrm{k}}\right)\right)$ and the boundedness of $\left\{u_{\varepsilon}\right\}_{\varepsilon}$ in $L^{p}\left(\Omega ; \mathbb{R}^{\mathrm{k}}\right)$. 
The next two propositions characterize the relationship between the $\boldsymbol{R}$-two-scale limit and the usual weak and strong limits in $L^{p}\left(\Omega ; \mathbb{R}^{\mathrm{k}}\right)$. These two results are simple adaptations of [44, Theorems 9 and 10] concerning the periodic case (also see [8, Proposition 2.10] concerning the $p=2$ case), which we include here for completeness.

Proposition 3.4. Assume that $\left\{u_{\varepsilon}\right\}_{\varepsilon} \subset L^{p}\left(\Omega ; \mathbb{R}^{\mathbb{k}}\right)$ is a sequence that $\boldsymbol{R}$-twoscale converges to a function $u \in L^{p}\left(\Omega \times Y^{\mathrm{m}} ; \mathbb{R}^{\mathbb{k}}\right)$. Then, $u_{\varepsilon} \rightarrow \bar{u}_{0}$ weakly in $L^{p}\left(\Omega ; \mathbb{R}^{\mathrm{k}}\right)$, where $\bar{u}_{0}(\cdot):=f_{Y^{\mathrm{m}}} u(\cdot, y) \mathrm{d} y$. In particular, $\left\{u_{\varepsilon}\right\}_{\varepsilon}$ is bounded in $L^{p}\left(\Omega ; \mathbb{R}^{\mathrm{k}}\right)$.

Proof. Let $\phi \in L^{p^{\prime}}\left(\Omega ; \mathbb{R}^{\mathrm{k}}\right)$, and set $\varphi(x, y):=\phi(x)$ for $(x, y) \in \Omega \times Y^{\mathrm{m}}$. Then, $\varphi \in L^{p^{\prime}}\left(\Omega ; C_{\#}\left(Y^{\mathrm{m}} ; \mathbb{R}^{\mathrm{k}}\right)\right)$, and by $(3.1)$ we have

$$
\begin{aligned}
\lim _{\varepsilon \rightarrow 0^{+}} \int_{\Omega} u_{\varepsilon}(x) \cdot \phi(x) \mathrm{d} x & =\lim _{\varepsilon \rightarrow 0^{+}} \int_{\Omega} u_{\varepsilon}(x) \cdot \varphi\left(x, \frac{\boldsymbol{R} x}{\varepsilon}\right) \mathrm{d} x \\
& =\int_{\Omega} f_{Y^{\mathrm{m}}} u(x, y) \cdot \varphi(x, y) \mathrm{d} x \mathrm{~d} y \\
& =\int_{\Omega}\left(f_{Y^{\mathrm{m}}} u(x, y) \mathrm{d} y\right) \cdot \phi(x) \mathrm{d} x
\end{aligned}
$$

and this concludes the proof.

Proposition 3.5. Let $\left\{u_{\varepsilon}\right\}_{\varepsilon} \subset L^{p}\left(\Omega ; \mathbb{R}^{\mathrm{k}}\right)$ and $u \in L^{p}\left(\Omega ; \mathbb{R}^{\mathrm{k}}\right)$ be such that $u_{\varepsilon} \rightarrow$ $u$ in $L^{p}\left(\Omega ; \mathbb{R}^{\mathrm{k}}\right)$ as $\varepsilon \rightarrow 0^{+}$. Then, $u_{\varepsilon} \stackrel{\boldsymbol{R}-2 s c}{\longrightarrow} u$.

Proof. Let $\varphi \in L^{p^{\prime}}\left(\Omega ; C_{\#}\left(Y^{\mathrm{m}} ; \mathbb{R}^{\mathrm{k}}\right)\right)$. Using Hölder's inequality, the convergence $u_{\varepsilon} \rightarrow u$ in $L^{p}\left(\Omega ; \mathbb{R}^{\mathbb{k}}\right)$, and Proposition 3.7 applied to $\psi(x, y):=u(x) \cdot \varphi(x, y)$, we get

$$
\begin{aligned}
\limsup _{\varepsilon \rightarrow 0^{+}}\left|\int_{\Omega} u_{\varepsilon}(x) \cdot \varphi\left(x, \frac{\boldsymbol{R} x}{\varepsilon}\right) \mathrm{d} x-\int_{\Omega} f_{Y^{\mathrm{m}}} u(x) \cdot \varphi(x, y) \mathrm{d} x \mathrm{~d} y\right| \\
\leqslant \limsup _{\varepsilon \rightarrow 0^{+}}\left(\left\|u_{\varepsilon}-u\right\|_{L^{p}\left(\Omega ; \mathbb{R}^{\mathrm{k}}\right)}\|\varphi\|_{L^{p^{\prime}}\left(\Omega ; C_{\#}\left(Y^{\mathrm{m}} ; \mathbb{R}^{\mathrm{k}}\right)\right)}\right. \\
\left.\quad+\left|\int_{\Omega} u(x) \cdot \varphi\left(x, \frac{\boldsymbol{R} x}{\varepsilon}\right) \mathrm{d} x-\int_{\Omega} f_{Y^{\mathrm{m}}} u(x) \cdot \varphi(x, y) \mathrm{d} x \mathrm{~d} y\right|\right)=0 .
\end{aligned}
$$

The proof of the following version of the Riemann-Lebesgue lemma may be found in [8, Lemma 2.4]. This lemma will be used in the subsequent proposition, which encodes nontrivial examples of sequences that $\boldsymbol{R}$-two-scale converge.

Lemma 3.6 (cf. [8, Lemma 2.4]). Let $\phi \in C_{\#}\left(Y^{\mathrm{m}} ; \mathbb{R}^{\mathrm{k}}\right)$, and assume that $\boldsymbol{R}$ satisfies (1.2). Then, the sequence $\left\{\phi_{\varepsilon}\right\}_{\varepsilon} \subset L^{\infty}\left(\Omega ; \mathbb{R}^{\mathrm{k}}\right)$ defined by $\phi_{\varepsilon}(x):=\phi\left(\frac{\boldsymbol{R} x}{\varepsilon}\right)$, $x \in \Omega$, converges weakly- $\star$ in $L^{\infty}\left(\Omega ; \mathbb{R}^{\mathrm{k}}\right)$ to the constant function $\bar{\phi}:=f_{Y^{\mathrm{m}}} \phi(y) \mathrm{d} y$.

As we mentioned above, the next proposition provides nontrivial examples of sequences that $\boldsymbol{R}$-two-scale converge; moreover, it will be useful to prove compactness of bounded sequences in $L^{p}\left(\Omega ; \mathbb{R}^{\mathrm{k}}\right)$ with respect to the $\boldsymbol{R}$-two-scale convergence. The proof is similar to that of [44, Lemma 2.5] concerning the periodic case, which we include here for completeness.

Proposition 3.7. Let $\psi \in L^{1}\left(\Omega ; C_{\#}\left(Y^{\mathrm{m}} ; \mathbb{R}^{\mathrm{k}}\right)\right)$, and assume that $\boldsymbol{R}$ satisfies (1.2). Then $\left\{\psi\left(\cdot, \frac{\boldsymbol{R} \cdot}{\varepsilon}\right)\right\}_{\varepsilon}$ is an equi-integrable sequence in $L^{1}\left(\Omega ; \mathbb{R}^{\mathbb{k}}\right)$ such that

$$
\left\|\psi\left(\cdot, \frac{\boldsymbol{R} \cdot}{\varepsilon}\right)\right\|_{L^{1}\left(\Omega ; \mathbb{R}^{\mathrm{k}}\right)} \leqslant\|\psi\|_{L^{1}\left(\Omega ; C_{\#}\left(Y^{\mathrm{m}} ; \mathbb{R}^{\mathrm{k}}\right)\right)}=\int_{\Omega} \sup _{y \in Y^{\mathrm{m}}}|\psi(x, y)| \mathrm{d} x
$$

Copyright $@$ by SIAM. Unauthorized reproduction of this article is prohibited. 
and

$$
\lim _{\varepsilon \rightarrow 0^{+}} \int_{\Omega} \psi\left(x, \frac{\boldsymbol{R} x}{\varepsilon}\right) \mathrm{d} x=\int_{\Omega} f_{Y^{\mathrm{m}}} \psi(x, y) \mathrm{d} x \mathrm{~d} y .
$$

Proof. The proof of (3.2) is immediate. Using this estimate (that holds with $\Omega$ replaced by any measurable set) and the integrability of the map $x \in \Omega \mapsto$ $\sup _{y \in Y^{\mathrm{m}}}|\psi(x, y)|$, we conclude that $\left\{\psi\left(\cdot, \frac{\boldsymbol{R}(\cdot)}{\varepsilon}\right)\right\}$ is equi-integrable in $L^{1}\left(\Omega ; \mathbb{R}^{\mathrm{k}}\right)$. Finally, the proof of (3.3) follows along the lines of that of [44, Lemma 2.5], which we detail next.

Step 1. Assume that $\psi$ is of the form $\psi(x, y)=\varphi(x) \phi(y)$ with $\varphi \in L^{1}(\Omega)$ and $\phi \in C_{\#}\left(Y^{\mathrm{m}} ; \mathbb{R}^{\mathrm{k}}\right)$. Then, (3.3) follows from Lemma 3.6.

Step 2. Assume that $\psi$ is of the form $\psi(x, y)=\sum_{k=1}^{j} c_{k} \chi_{A_{k}}(x) \phi_{k}(y)$, where $j \in \mathbb{N}, c_{k}$ are distinct real numbers, $A_{k}$ are mutually disjoint measurable subsets of $\Omega$, and $\phi_{k} \in C_{\#}\left(Y^{\mathrm{m}} ; \mathbb{R}^{\mathrm{k}}\right)$. Then, (3.3) follows from Step 1 .

Step 3. Let $\psi \in L^{1}\left(\Omega ; C_{\#}\left(Y^{\mathrm{m}} ; \mathbb{R}^{\mathrm{k}}\right)\right)$. We can find a sequence $\left\{\psi_{j}\right\}_{j \in \mathbb{N}}$ of step functions as in Step 2 such that $\psi_{j} \rightarrow \psi$ in $L^{1}\left(\Omega ; C_{\#}\left(Y^{\mathrm{m}} ; \mathbb{R}^{\mathrm{k}}\right)\right)$ as $j \rightarrow \infty$. Fix $j \in \mathbb{N}$; in view of (3.2), we have

$$
\begin{aligned}
& \left|\int_{\Omega} \psi\left(x, \frac{\boldsymbol{R} x}{\varepsilon}\right) \mathrm{d} x-\int_{\Omega} f_{Y^{\mathrm{m}}} \psi(x, y) \mathrm{d} x \mathrm{~d} y\right| \\
& \leqslant \int_{\Omega}\left|\psi\left(x, \frac{\boldsymbol{R} x}{\varepsilon}\right)-\psi_{j}\left(x, \frac{\boldsymbol{R} x}{\varepsilon}\right)\right| \mathrm{d} x+\left|\int_{\Omega} \psi_{j}\left(x, \frac{\boldsymbol{R} x}{\varepsilon}\right) \mathrm{d} x-\int_{\Omega} f_{Y^{\mathrm{m}}} \psi_{j}(x, y) \mathrm{d} x \mathrm{~d} y\right| \\
& \quad+\int_{\Omega} f_{Y^{\mathrm{m}}}\left|\psi_{j}(x, y)-\psi(x, y)\right| \mathrm{d} x \mathrm{~d} y \\
& \leqslant \\
& \quad\left(1+\left[\mathcal{L}^{\mathrm{m}}\left(Y^{\mathrm{m}}\right)\right]^{-1}\right)\left\|\psi-\psi_{j}\right\|_{L^{1}\left(\Omega ; C_{\#}\left(Y^{\mathrm{m}} ; \mathbb{R}^{\mathrm{k}}\right)\right)} \\
& \quad+\left|\int_{\Omega} \psi_{j}\left(x, \frac{\boldsymbol{R} x}{\varepsilon}\right) \mathrm{d} x-\int_{\Omega} f_{Y^{\mathrm{m}}} \psi_{j}(x, y) \mathrm{d} x \mathrm{~d} y\right| .
\end{aligned}
$$

Letting $\varepsilon \rightarrow 0^{+}$and using Step 2 first, and then letting $j \rightarrow \infty$, we obtain (3.3) from the convergence $\psi_{j} \rightarrow \psi$ in $L^{1}\left(\Omega ; C_{\#}\left(Y^{\mathrm{m}} ; \mathbb{R}^{\mathrm{k}}\right)\right)$ as $j \rightarrow \infty$.

Corollary 3.8. Let $\psi \in L^{p}\left(\Omega ; C_{\#}\left(Y^{\mathrm{m}} ; \mathbb{R}^{\mathrm{k}}\right)\right)$, and assume that $\boldsymbol{R}$ satisfies $(1.2)$. Then, $\left\{\psi\left(\cdot, \frac{\boldsymbol{R} \cdot}{\varepsilon}\right)\right\}_{\varepsilon}$ is a p-equi-integrable sequence in $L^{p}\left(\Omega ; \mathbb{R}^{\mathrm{k}}\right)$ that $\boldsymbol{R}$-two-scale converges to $\psi$.

Proof. The p-equi-integrability assertion follows from Proposition 3.7 applied to $|\psi|^{p}$. The $\boldsymbol{R}$-two-scale convergence assertion follows from (3.3) with $\psi$ replaced by $\psi \varphi$, where $\varphi \in L^{p^{\prime}}\left(\Omega ; C_{\#}\left(Y^{\mathrm{m}} ; \mathbb{R}^{\mathrm{k}}\right)\right)$ is an arbitrary function.

Using the previous proposition, we establish next a compactness property with respect to the $\boldsymbol{R}$-two-scale convergence. The proof follows along the lines of that of [44, Theorem 14] in the context of the periodic case, which we include here for completeness.

Proposition 3.9. Let $\left\{u_{\varepsilon}\right\}_{\varepsilon} \subset L^{p}\left(\Omega ; \mathbb{R}^{\mathrm{k}}\right)$ be a bounded sequence, and assume that $\boldsymbol{R}$ satisfies (1.2). Then, there exist a subsequence $\varepsilon^{\prime} \preceq \varepsilon$ and a function $u \in$ $L^{p}\left(\Omega \times Y^{\mathrm{m}} ; \mathbb{R}^{\mathrm{k}}\right)$ such that $u_{\varepsilon^{\prime}} \stackrel{\boldsymbol{R}-2 s c}{\longrightarrow} u$.

Proof. To simplify the notation, set $X:=L^{p^{\prime}}\left(\Omega ; C_{\#}\left(Y^{\mathrm{m}} ; \mathbb{R}^{\mathrm{k}}\right)\right)$, and denote by $X^{\prime}$ the dual of $X$. Let $L_{\varepsilon}: X \rightarrow \mathbb{R}$ be the linear map defined, for $\varphi \in X$, by

$$
L_{\varepsilon}(\varphi):=\int_{\Omega} u_{\varepsilon}(x) \cdot \varphi\left(x, \frac{\boldsymbol{R} x}{\varepsilon}\right) \mathrm{d} x .
$$

Copyright (c) by SIAM. Unauthorized reproduction of this article is prohibited. 
By Hölder's inequality, we have $\left|L_{\varepsilon}(\varphi)\right| \leqslant c\|\varphi\|_{X}$, where $c:=\sup _{\varepsilon}\left\|u_{\varepsilon}\right\|_{L^{p}\left(\Omega ; \mathbb{R}^{\mathrm{k}}\right)}$. Thus, by the Riesz representation theorem, there exists $U_{\varepsilon} \in X^{\prime}$ such that $\left\langle U_{\varepsilon}, \varphi\right\rangle_{X^{\prime}, X}=$ $L_{\varepsilon}(\varphi)$ for all $\varphi \in X$. Next, we observe that $X$ is separable and

$$
\left(\left\|U_{\varepsilon}\right\|_{X^{\prime}}=\sup _{\varphi \in X,\|\varphi\|_{X} \leqslant 1}\left|\left\langle U_{\varepsilon}, \varphi\right\rangle_{X^{\prime}, X}\right| \leqslant c\right) .
$$

Hence, by the Alaoglu theorem, there exist a subsequence $\varepsilon^{\prime} \preceq \varepsilon$ and a function $U \in X^{\prime}$ such that $\lim _{\varepsilon^{\prime} \rightarrow 0^{+}}\left\langle U_{\varepsilon^{\prime}}, \varphi\right\rangle_{X^{\prime}, X}=\langle U, \varphi\rangle_{X^{\prime}, X}$ for all $\varphi \in X$. Passing the inequality

$$
\left|\left\langle U_{\varepsilon^{\prime}}, \varphi\right\rangle_{X^{\prime}, X}\right|=\left|L_{\varepsilon^{\prime}}(\varphi)\right| \leqslant c\left(\int_{\Omega}\left|\varphi\left(x, \frac{\boldsymbol{R} x}{\varepsilon^{\prime}}\right)\right|^{p^{\prime}} \mathrm{d} x\right)^{\frac{1}{p^{\prime}}}
$$

to the limit as $\varepsilon^{\prime} \rightarrow 0^{+}$, and invoking Proposition 3.7 applied to $\psi(x, y):=|\varphi(x, y)|^{p^{\prime}}$, we obtain

$$
\left|\langle U, \varphi\rangle_{X^{\prime}, X}\right| \leqslant c\left(\int_{\Omega} f_{Y^{\mathrm{m}}}|\varphi(x, y)|^{p^{\prime}} \mathrm{d} x \mathrm{~d} y\right)^{\frac{1}{p^{\prime}}}=c\left[\mathcal{L}^{\mathrm{m}}\left(Y^{\mathrm{m}}\right)\right]^{-\frac{1}{p^{\prime}}}\|\varphi\|_{L^{p^{\prime}}\left(\Omega \times Y^{\mathrm{m}} ; \mathbb{R}^{\mathrm{k}}\right)}
$$

for all $\varphi \in X$. Finally, using the density of $X$ in $L^{p^{\prime}}\left(\Omega \times Y^{\mathrm{m}} ; \mathbb{R}^{\mathbb{k}}\right)$, we see that $U$ can be continuously extended to $L^{p^{\prime}}\left(\Omega \times Y^{\mathrm{m}} ; \mathbb{R}^{\mathrm{k}}\right)$ with $(3.4)$ valid for all $\varphi \in L^{p^{\prime}}\left(\Omega \times Y^{\mathrm{m}} ; \mathbb{R}^{\mathrm{k}}\right)$. Consequently, by the Riesz representation theorem there exists $\bar{u} \in L^{p}\left(\Omega \times Y^{\mathrm{m}} ; \mathbb{R}^{\mathrm{k}}\right)$ such that, for all $\varphi \in L^{p^{\prime}}\left(\Omega \times Y^{\mathrm{m}} ; \mathbb{R}^{\mathrm{k}}\right)$,

$$
\langle U, \varphi\rangle_{X^{\prime}, X}=\int_{\Omega} \int_{Y^{\mathrm{m}}} \bar{u}(x, y) \cdot \varphi(x, y) \mathrm{d} x \mathrm{~d} y .
$$

In particular, this last identity holds for all $\varphi \in X$, from which we conclude the proof by taking $u:=\mathcal{L}^{\mathrm{m}}\left(Y^{\mathrm{m}}\right) \bar{u}$.

Remark 3.10. As shown in [8, Remark 2.8], Proposition 3.9 may fail if there exists $k \in \mathbb{Z}^{\mathrm{m}} \backslash\{0\}$ such that $\boldsymbol{R}^{*} k=0$.

3.1. $R$-two-scale limits of $\mathcal{A}$-free sequences. In this subsection, we characterize the $\boldsymbol{R}$-two-scale limits associated with $L^{p}$-bounded sequences of $\mathcal{A}$-free vector fields, as stated in Theorem 1.3. As we will show, this characterization is intimately related to the notion of $\left(\mathcal{A}, \mathcal{A}_{\boldsymbol{R}^{*}}^{y}\right)$-free vector fields introduced below.

Definition $3.11\left(\mathcal{A}_{\boldsymbol{R}^{*}}\right.$ and $\mathcal{A}_{\boldsymbol{R}^{*}}^{y}$-free fields). We say that $v \in L_{\#}^{p}\left(Y^{\mathrm{m}} ; \mathbb{R}^{\mathrm{d}}\right)$ is $\mathcal{A}_{\boldsymbol{R}^{*}}$-free, and write $\mathcal{A}_{\boldsymbol{R}^{*}} v=0$, if for all $\psi \in C_{\#}^{1}\left(Y^{\mathrm{m}} ; \mathbb{R}^{\mathbb{1}}\right)$, we have

$$
\int_{Y^{\mathrm{m}}} v(y) \cdot \mathcal{A}_{\boldsymbol{R}^{*}}^{*} \psi(y) \mathrm{d} y=0
$$

where

$$
\mathcal{A}_{\boldsymbol{R}}^{*}:=-\sum_{i=1}^{\mathrm{m}} \sum_{m=1}^{\mathrm{m}}\left(A^{(i)}\right)^{T} \boldsymbol{R}_{m i} \frac{\partial}{\partial y_{m}} .
$$

We say that $w \in L^{p}\left(\Omega ; L_{\#}^{p}\left(Y^{\mathrm{m}} ; \mathbb{R}^{\mathrm{d}}\right)\right)$, with $w=w(x, y)$, is $\mathcal{A}_{\boldsymbol{R}^{*}}^{y}$-free, and write $\mathcal{A}_{\boldsymbol{R}^{*}}^{y} w=0$, if $\mathcal{A}_{\boldsymbol{R}^{*}} w(x, \cdot)=0$ for a.e. $x \in \Omega$.

Copyright (c) by SIAM. Unauthorized reproduction of this article is prohibited. 


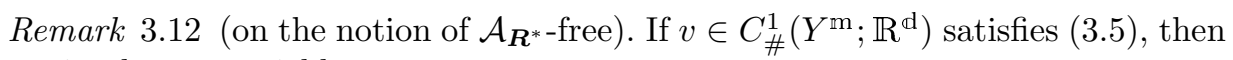
integration by parts yields

$$
\begin{aligned}
0 & =\int_{Y^{\mathrm{m}}} v(y) \cdot \mathcal{A}_{\boldsymbol{R}}^{*} \psi(y) \mathrm{d} y=-\int_{Y^{\mathrm{m}}} v(y) \cdot \sum_{i=1}^{\mathrm{m}} \sum_{m=1}^{\mathrm{m}}\left(A^{(i)}\right)^{T} \boldsymbol{R}_{m i} \frac{\partial \psi}{\partial y_{m}}(y) \mathrm{d} y \\
& =-\int_{Y^{\mathrm{m}}} \sum_{i=1}^{\mathrm{m}} \sum_{m=1}^{\mathrm{m}} \boldsymbol{R}_{i m}^{*} A^{(i)} v(y) \cdot \frac{\partial \psi}{\partial y_{m}}(y) \mathrm{d} y=\int_{Y^{\mathrm{m}}} \sum_{i=1}^{\mathrm{m}} \sum_{m=1}^{\mathrm{m}} \boldsymbol{R}_{i m}^{*} A^{(i)} \frac{\partial v}{\partial y_{m}}(y) \cdot \psi(y) \mathrm{d} y
\end{aligned}
$$

for all $\psi \in C_{\#}^{1}\left(Y^{\mathrm{m}} ; \mathbb{R}^{\mathbb{1}}\right)$. Thus, $\mathcal{A}_{\boldsymbol{R}^{*}} v=0$ pointwise in $\mathbb{R}^{\mathrm{m}}$, where

$$
\stackrel{\circ}{A}_{\boldsymbol{R}^{*}}:=-\sum_{i=1}^{\mathrm{m}} \sum_{m=1}^{\mathrm{m}} \boldsymbol{R}_{i m}^{*} A^{(i)} \frac{\partial}{\partial y_{m}} .
$$

We observe further that, as a consequence of our analysis in section 5 (see Remark 5.7), in $\mathbb{R}^{\mathrm{m}}$ and in the $\mathcal{A}=$ curl case we have that $v \in L_{\#}^{p}\left(Y^{\mathrm{m}} ; \mathbb{R}^{\mathrm{m}}\right)$ is $\mathcal{A}_{\boldsymbol{R}^{*}}$-free if and only if $v \in \mathcal{G}_{\boldsymbol{R}}^{p}$, where $\mathcal{G}_{\boldsymbol{R}}^{p}$ is given by (1.12).

Definition $3.13\left(\left(\mathcal{A}, \mathcal{A}_{\boldsymbol{R}^{*}}^{y}\right)\right.$-free fields). Let $w \in L^{p}\left(\Omega ; L_{\#}^{p}\left(Y^{\mathrm{m}} ; \mathbb{R}^{\mathrm{d}}\right)\right)$, and define $\bar{w}_{0} \in L^{p}\left(\Omega ; \mathbb{R}^{\mathrm{d}}\right)$ and $\bar{w}_{1} \in L^{p}\left(\Omega ; L_{\#}^{p}\left(Y^{\mathrm{m}} ; \mathbb{R}^{\mathrm{d}}\right)\right)$ by setting $\bar{w}_{0}:=f_{Y^{\mathrm{m}}} w(\cdot, y) \mathrm{d} y$ and $\bar{w}_{1}:=w-\bar{w}_{0}$. We say that $w$ is $\left(\mathcal{A}, \mathcal{A}_{\boldsymbol{R}^{*}}^{y}\right)$-free if

$$
\mathcal{A} \bar{w}_{0}=0 \quad \text { and } \quad \mathcal{A}_{\boldsymbol{R}^{*}}^{y} \bar{w}_{1}=0
$$

in the sense of Definitions 2.1 and 3.11, respectively.

The next proposition shows that the $\boldsymbol{R}$-two-scale limit of an $L^{p}$-bounded sequence of $\mathcal{A}$-free vector fields is necessarily $\left(\mathcal{A}, \mathcal{A}_{\boldsymbol{R}^{*}}^{y}\right)$-free. This result is the quasi-periodic counterpart of [31, Proposition 2.11] concerning the periodic case.

Proposition 3.14. Let $\left\{u_{\varepsilon}\right\}_{\varepsilon}$ be a bounded and $\mathcal{A}$-free sequence in $L^{p}\left(\Omega ; \mathbb{R}^{\mathbb{d}}\right)$. Assume that there exists a function $u \in L^{p}\left(\Omega \times Y^{\mathrm{m}} ; \mathbb{R}^{\mathrm{d}}\right)$ such that $u_{\varepsilon} \stackrel{\boldsymbol{R}-2 s c}{\longrightarrow} u$. Then, $u$ is $\left(\mathcal{A}, \mathcal{A}_{\boldsymbol{R}^{*}}^{y}\right)$-free in the sense of Definition 3.13 .

Proof. Let $\phi \in C_{c}^{1}\left(\Omega ; \mathbb{R}^{\mathbb{1}}\right)$. Using the fact that each $u_{\varepsilon}$ is $\mathcal{A}$-free first, and invoking (3.1) applied to $\varphi:=\mathcal{A}^{*} \phi$, we get

$0=\lim _{\varepsilon \rightarrow 0^{+}} \int_{\Omega} u_{\varepsilon}(x) \cdot \mathcal{A}^{*} \phi(x) \mathrm{d} x=\int_{\Omega} f_{Y^{\mathrm{m}}} u(x, y) \cdot \mathcal{A}^{*} \phi(x) \mathrm{d} x \mathrm{~d} y=\int_{\Omega} \bar{u}_{0}(x) \cdot \mathcal{A}^{*} \phi(x) \mathrm{d} x$,

where $\bar{u}_{0}:=f_{Y^{\mathrm{m}}} u(\cdot, y) \mathrm{d} y$. Recalling Definition 2.1, (3.6) shows that $\mathcal{A} \bar{u}_{0}=0$ in $L^{p}\left(\Omega ; \mathbb{R}^{\mathbb{1}}\right)$.

Next, we prove that $\mathcal{A}_{\boldsymbol{R}^{*}}^{y} \bar{u}_{1}=0$ with $\bar{u}_{1}:=u-\bar{u}_{0}$. Let $\phi \in C_{c}^{1}(\Omega)$ and $\psi \in$ $C_{\#}^{1}\left(Y^{\mathrm{m}} ; \mathbb{R}^{\mathbb{1}}\right)$, and set $\varphi_{\varepsilon}(x):=\varepsilon \phi(x) \psi\left(\frac{\boldsymbol{R} x}{\varepsilon}\right)$ for $x \in \Omega$. Then $\varphi_{\varepsilon} \in C_{c}^{1}\left(\Omega ; \mathbb{R}^{\mathbb{1}}\right)$ with

$$
\begin{aligned}
\mathcal{A}^{*} \varphi_{\varepsilon}(x) & =-\sum_{i=1}^{\mathrm{m}}\left(A^{(i)}\right)^{T} \frac{\partial \varphi_{\varepsilon}}{\partial x_{i}}(x) \\
& =-\varepsilon \sum_{i=1}^{\mathrm{m}} \frac{\partial \phi}{\partial x_{i}}(x)\left(A^{(i)}\right)^{T} \psi\left(\frac{\boldsymbol{R} x}{\varepsilon}\right)-\phi(x) \sum_{i=1}^{\mathrm{m}}\left[\left(A^{(i)}\right)^{T} \sum_{m=1}^{\mathrm{m}} \frac{\partial \psi}{\partial y_{m}}\left(\frac{\boldsymbol{R} x}{\varepsilon}\right) \boldsymbol{R}_{m i}\right] \\
& =-\varepsilon \sum_{i=1}^{\mathrm{n}} \frac{\partial \phi}{\partial x_{i}}(x)\left(A^{(i)}\right)^{T} \psi\left(\frac{\boldsymbol{R} x}{\varepsilon}\right)-\phi(x) \sum_{i=1}^{\mathrm{m}} \sum_{m=1}^{\mathrm{m}}\left(A^{(i)}\right)^{T} \boldsymbol{R}_{m i} \frac{\partial \psi}{\partial y_{m}}\left(\frac{\boldsymbol{R} x}{\varepsilon}\right) .
\end{aligned}
$$

Copyright (c) by SIAM. Unauthorized reproduction of this article is prohibited. 
Hence, arguing as above, we have

$$
\begin{aligned}
0= & \lim _{\varepsilon \rightarrow 0^{+}} \int_{\Omega} u_{\varepsilon}(x) \mathcal{A}^{*} \varphi_{\varepsilon}(x) \mathrm{d} x \\
= & \lim _{\varepsilon \rightarrow 0^{+}} \int_{\Omega} u_{\varepsilon}(x) \cdot\left(-\varepsilon \sum_{i=1}^{\mathrm{m}} \frac{\partial \phi}{\partial x_{i}}(x)\left(A^{(i)}\right)^{T} \psi\left(\frac{\boldsymbol{R} x}{\varepsilon}\right)\right. \\
& \left.-\phi(x) \sum_{i=1}^{\mathrm{m}} \sum_{m=1}^{\mathrm{m}}\left(A^{(i)}\right)^{T} \boldsymbol{R}_{m i} \frac{\partial \psi}{\partial y_{m}}\left(\frac{\boldsymbol{R} x}{\varepsilon}\right)\right) \mathrm{d} x \\
= & -\int_{\Omega} f_{Y^{\mathrm{m}}} u(x, y) \cdot\left(\phi(x) \sum_{i=1}^{\mathrm{m}} \sum_{m=1}^{\mathrm{m}}\left(A^{(i)}\right)^{T} \boldsymbol{R}_{m i} \frac{\partial \psi}{\partial y_{m}}(y)\right) \mathrm{d} x \mathrm{~d} y \\
= & -\int_{\Omega} f_{Y^{\mathrm{m}}} u(x, y) \cdot \phi(x) \mathcal{A}_{\boldsymbol{R}}^{*} \psi(y) \mathrm{d} x \mathrm{~d} y=-\int_{\Omega} f_{Y^{\mathrm{m}}} \bar{u}_{1}(x, y) \cdot \phi(x) \mathcal{A}_{\boldsymbol{R}}^{*} \psi(y) \mathrm{d} x \mathrm{~d} y,
\end{aligned}
$$

where in the last equality we used the fact that $\bar{u}_{0}$ depends only on $x$, and $\int_{Y^{\mathrm{m}}} \mathcal{A}_{\boldsymbol{R}}^{*} \psi(y) \mathrm{d} y=$ 0 by the periodicity of $\psi$.

Because (3.7) holds for all $\phi \in C_{c}^{1}(\Omega)$ and $\psi \in C_{\#}^{1}\left(Y^{\mathrm{m}} ; \mathbb{R}^{\mathbb{1}}\right)$ and $C_{\#}^{1}\left(Y^{\mathrm{m}} ; \mathbb{R}^{\mathbb{1}}\right)$ is separable, we conclude that $\mathcal{A}_{\boldsymbol{R}^{*}}^{y} \bar{u}_{1}=0$ in the sense of Definition 3.11.

The next proposition shows that Proposition 3.14 fully characterizes the $\boldsymbol{R}$-twoscale limit of an $L^{p}$-bounded sequence of $\mathcal{A}$-free vector fields, as we prove that any $\left(\mathcal{A}, \mathcal{A}_{\boldsymbol{R}^{*}}^{y}\right)$-free vector field is attained as the $\boldsymbol{R}$-two-scale limit of such a sequence. This result is the quasi-periodic counterpart of [31, Proposition 2.11] concerning the periodic case. Moreover, as we mentioned in the introduction, this result is new in the literature even for $p=2$ and $\mathcal{A}=\operatorname{curl}$ or $\mathcal{A}=\operatorname{div}$ which were treated in $[8,60]$.

Proposition 3.15. Let $u \in L^{p}\left(\Omega ; L_{\#}^{p}\left(Y^{\mathrm{m}} ; \mathbb{R}^{\mathrm{d}}\right)\right)$ be an $\left(\mathcal{A}, \mathcal{A}_{\boldsymbol{R}^{*}}^{y}\right)$-free vector field in the sense of Definition 3.13, and assume that $\boldsymbol{R}$ satisfies (1.2). Then, there exists a bounded and $\mathcal{A}$-free sequence, $\left\{u_{\varepsilon}\right\}_{\varepsilon}$, in $L^{p}\left(\Omega ; \mathbb{R}^{\mathrm{d}}\right)$ such that $u_{\varepsilon} \stackrel{\boldsymbol{R} \text {-2sc }}{\longrightarrow} u$.

Proof. Fix $u \in L^{p}\left(\Omega ; L_{\#}^{p}\left(Y^{\mathrm{m}} ; \mathbb{R}^{\mathrm{d}}\right)\right)$, an $\left(\mathcal{A}, \mathcal{A}_{\boldsymbol{R}^{*}}^{y}\right)$-free vector field. We have

$$
\mathcal{A} \bar{u}_{0}=0 \quad \text { and } \quad \mathcal{A}_{\boldsymbol{R}^{*}}^{y} \bar{u}_{1}=0
$$

in the sense of Definition 2.1 and Definition 3.11, respectively, where $\bar{u}_{0}:=f_{Y^{\mathrm{m}}} u(\cdot, y) \mathrm{d} y$ and $\bar{u}_{1}:=u-\bar{u}_{0}$. Note that for a.e. $x \in \Omega$, it holds that

$$
\int_{Y^{\mathrm{m}}} \bar{u}_{1}(x, y) \mathrm{d} y=0
$$

We will proceed in three steps.

Step 1. Assume that $\bar{u}_{0}=0$ and $\bar{u}_{1} \in C_{c}^{1}\left(\mathbb{R}^{\mathrm{n}} ; C_{\#}^{1}\left(Y^{\mathrm{m}} ; \mathbb{R}^{\mathrm{d}}\right)\right)$. In this case, (3.9) holds for all $x \in \Omega$, and, as observed in Remark 3.12, we have

$$
\mathcal{A}_{\boldsymbol{R}^{*}} \bar{u}_{1}=0 \text { pointwise in } \Omega \times \mathbb{R}^{\mathrm{m}}, \text { where } \mathcal{A}_{\boldsymbol{R}^{*}}=-\sum_{i=1}^{\mathrm{m}} \sum_{m=1}^{\mathrm{m}} \boldsymbol{R}_{i m}^{*} A^{(i)} \frac{\partial}{\partial y_{m}} .
$$

For each $\varepsilon>0$, define $v_{\varepsilon} \in C_{c}^{1}\left(\mathbb{R}^{\mathrm{m}}\right)$ by setting

$$
v_{\varepsilon}(x):=\bar{u}_{1}\left(x, \frac{\boldsymbol{R} x}{\varepsilon}\right) \text { for } x \in \mathbb{R}^{\mathrm{n}} .
$$

Copyright (C) by SIAM. Unauthorized reproduction of this article is prohibited. 
By Corollary 3.8 and Proposition 3.4, together with (3.9), we obtain

$$
\begin{aligned}
& \left\{v_{\varepsilon}\right\}_{\varepsilon} \text { is a } p \text {-equi-integrable sequence in } L^{p}\left(\Omega ; \mathbb{R}^{\mathrm{d}}\right), \\
& v_{\varepsilon} \stackrel{\boldsymbol{R}-2 s c}{\longrightarrow} \bar{u}_{1}, \\
& v_{\varepsilon} \rightarrow 0 \text { weakly in } L^{p}\left(\Omega ; \mathbb{R}^{\mathrm{d}}\right) .
\end{aligned}
$$

On the other hand, in view of (3.10) and recalling Remark 2.2, we have

$$
\mathcal{A} v_{\varepsilon}(x)=\left(\mathcal{A}_{x} \bar{u}_{1}\right)\left(x, \frac{\boldsymbol{R} x}{\varepsilon}\right)
$$

for all $x \in \Omega$. Because $\mathcal{A}_{x} \bar{u}_{1} \in C_{c}\left(\mathbb{R}^{\mathrm{n}} ; C_{\#}^{1}\left(Y^{\mathrm{m}} ; \mathbb{R}^{\mathbb{1}}\right)\right)$, we may invoke Proposition 3.4 and (3.9) once more to conclude that

$$
\mathcal{A} v_{\varepsilon} \rightarrow f_{Y^{\mathrm{m}}}\left(\mathcal{A}_{x} \bar{u}_{1}\right)(x, y) \mathrm{d} y=\mathcal{A}_{x}\left(f_{Y^{\mathrm{m}}} \bar{u}_{1}(x, y) \mathrm{d} y\right)=0 \text { weakly in } L^{p}\left(\Omega ; \mathbb{R}^{\mathbb{1}}\right) .
$$

Hence,

$$
\mathcal{A} v_{\varepsilon} \rightarrow 0 \text { in } W^{-1, p}\left(\Omega ; \mathbb{R}^{\mathbb{1}}\right) .
$$

Let $\Pi \subset \mathbb{R}^{\mathrm{m}}$ be a parallelotope containing $\Omega$. By Lemma 2.3, we can find a $p$ equi-integrable sequence in $\Pi,\left\{u_{\varepsilon}\right\}_{\varepsilon} \subset L^{p}\left(\Pi ; \mathbb{R}^{\mathbb{d}}\right)$, and a positive constant depending only on $\mathcal{A}, C=C(\mathcal{A})$, such that

$$
\begin{aligned}
& \mathcal{A} u_{\varepsilon}=0 \text { for all } \varepsilon>0, \\
& u_{\varepsilon}-v_{\varepsilon} \rightarrow 0 \text { in } L^{p}\left(\Omega ; \mathbb{R}^{\mathrm{d}}\right), \\
& \left\|u_{\varepsilon}\right\|_{L^{p}\left(\Pi ; \mathbb{R}^{\mathrm{d}}\right)} \leqslant C\left\|v_{\varepsilon}\right\|_{L^{p}\left(\Omega ; \mathbb{R}^{\mathrm{d}}\right)} \text { for all } \varepsilon>0 .
\end{aligned}
$$

To conclude Step 1, we observe that the second condition in (3.12) and (3.14), together with Proposition 3.5, yield

$$
u_{\varepsilon} \stackrel{R-2 s c}{\longrightarrow} \bar{u}_{1} .
$$

Step 2. Assume that $\bar{u}_{0}=0$ and $\bar{u}_{1} \in L^{p}\left(\Omega ; L_{\#}^{p}\left(Y^{\mathrm{m}} ; \mathbb{R}^{\mathrm{d}}\right)\right)$.

For all $y \in \mathbb{R}^{\mathrm{m}}$, we extend $\bar{u}_{1}(\cdot, y)$ by zero outside $\Omega$, which we still denote by $\bar{u}_{1}$. Let $\left\{\rho_{j}\right\}_{j \in \mathbb{N}} \subset C_{c}^{\infty}\left(\mathbb{R}^{\mathrm{m}}\right)$ and $\left\{\rho_{j}^{\#}\right\}_{j \in \mathbb{N}} \subset C_{\#}^{\infty}\left(Y^{\mathrm{m}}\right)$ be sequences of standard, symmetric mollifiers. For each $j \in \mathbb{N}$, we define

$$
\begin{aligned}
\tilde{u}_{j}(x, y) & :=\int_{\mathbb{R}^{\mathrm{n}}} \int_{Y^{\mathrm{m}}} \bar{u}_{1}\left(x^{\prime}, y^{\prime}\right) \rho_{j}\left(x-x^{\prime}\right) \rho_{j}^{\#}\left(y-y^{\prime}\right) \mathrm{d} x^{\prime} \mathrm{d} y^{\prime} \\
& =\int_{\mathbb{R}^{\mathrm{n}}} \int_{Y^{\mathrm{m}}} \bar{u}_{1}\left(x^{\prime}, y^{\prime}+y\right) \rho_{j}\left(x-x^{\prime}\right) \rho_{j}^{\#}\left(y^{\prime}\right) \mathrm{d} x^{\prime} \mathrm{d} y,
\end{aligned}
$$

where in the last equality we used the $Y^{\mathrm{m}}$-periodicity of $\bar{u}_{1}$ along with the symmetry and $Y^{\mathrm{m}}$-periodicity of $\rho_{j}^{\#}$. By standard mollification arguments, we have $\tilde{u}_{j} \in C_{c}^{\infty}\left(\mathbb{R}^{\mathrm{n}} ; C_{\#}^{\infty}\left(Y^{\mathrm{m}} ; \mathbb{R}^{\mathbb{d}}\right)\right)$ with

$$
\left\|\tilde{u}_{j}\right\|_{L^{p}\left(\mathbb{R}^{\mathrm{n}} ; L^{p}\left(Y^{\mathrm{m}}: \mathbb{R}^{\mathrm{d}}\right)\right)} \leqslant\left\|\bar{u}_{1}\right\|_{L^{p}\left(\Omega ; L^{p}\left(Y^{\mathrm{m}}: \mathbb{R}^{\mathrm{d}}\right)\right)} .
$$

Moreover,

$$
\mathcal{A}_{\boldsymbol{R}^{*}} \tilde{u}_{j}=0 \text { pointwise in } \Omega \times \mathbb{R}^{\mathrm{m}} \text { and } \int_{Y^{\mathrm{m}}} \tilde{u}_{j}(\cdot, y) \mathrm{d} y=0
$$

Copyright (c) by SIAM. Unauthorized reproduction of this article is prohibited. 
by (3.8) and (3.9), together with the $Y^{\mathrm{m}}$-periodicity of $\bar{u}_{1}$ and Fubini's theorem.

By Step 1, for each $j \in \mathbb{N}$, we can find a p-equi-integrable sequence in $\Pi,\left\{u_{\varepsilon}^{(j)}\right\} \subset$ $L^{p}\left(\Pi ; \mathbb{R}^{d}\right)$, satisfying (3.14)-(3.15) with $u_{\varepsilon}$ replaced by $u_{\varepsilon}^{(j)}$ and, recalling $(3.11), v_{\varepsilon}$ replaced by

$$
v_{\varepsilon}^{(j)}(x):=\tilde{u}_{j}\left(x, \frac{\boldsymbol{R} x}{\varepsilon}\right) \text { for } x \in \mathbb{R}^{\mathrm{n}} .
$$

In particular, we have

$$
\begin{aligned}
\limsup _{j \rightarrow \infty} \limsup _{\varepsilon \rightarrow 0^{+}} \int_{\Omega}\left|u_{\varepsilon}^{(j)}(x)\right|^{p} \mathrm{~d} x & \leqslant C^{p} \limsup _{j \rightarrow \infty} \limsup _{\varepsilon \rightarrow 0^{+}} \int_{\Omega}\left|\tilde{u}_{j}\left(x, \frac{\boldsymbol{R} x}{\varepsilon}\right)\right|^{p} \mathrm{~d} x \\
& =C^{p} \limsup _{j \rightarrow \infty} \int_{\Omega} f_{Y^{\mathrm{m}}}\left|\tilde{u}_{j}(x, y)\right|^{p} \mathrm{~d} x \mathrm{~d} y \\
& \leqslant \mathcal{L}^{\mathrm{m}}\left(Y^{\mathrm{m}}\right) C^{p}\left\|\bar{u}_{1}\right\|_{L^{p}\left(\Omega ; L^{p}\left(Y^{\mathrm{m}}: \mathbb{R}^{\mathrm{d}}\right)\right) .}
\end{aligned}
$$

This estimate and the separability of $L^{p^{\prime}}\left(\Omega ; C_{\#}\left(Y^{\mathrm{m}} ; \mathbb{R}^{\mathrm{d}}\right)\right)$ allow us to use a diagonalization argument as in [29, proof of Prop. 1.11, p. 449] to find a sequence $\left(j_{\varepsilon}\right)_{\varepsilon}$ such that $j_{\varepsilon} \rightarrow \infty$ as $\varepsilon \rightarrow 0^{+}$and $u_{\varepsilon}:=u_{\varepsilon}^{\left(j_{\varepsilon}\right)}$ satisfies the required properties.

Step 3 . We treat the general case.

By Step 2, there exists a bounded and $\mathcal{A}$-free sequence, $\left\{u_{\varepsilon}\right\}_{\varepsilon}$, in $L^{p}\left(\Omega ; \mathbb{R}^{\mathrm{d}}\right)$ such that $u_{\varepsilon} \stackrel{\boldsymbol{R} \text {-2sc }}{\longrightarrow} u_{1}$. Defining $\hat{u}_{\varepsilon}:=u_{0}+u_{\varepsilon}$, we have $\mathcal{A} \hat{u}_{\varepsilon}=0$ and $\hat{u}_{\varepsilon} \stackrel{\boldsymbol{R} \text {-2sc }}{\longrightarrow}$ $u_{0}+u_{1}=u$, using (3.8) and Proposition 3.5.

Proof of Theorem 1.3. The statement in Theorem 1.3 in an immediate consequence of Propositions 3.14 and 3.15 .

4. $\Gamma$-convergence homogenization. In this section, we prove Theorem 1.1. To this end, we first show in Theorem 4.1 below that the sequence $\left\{F_{\varepsilon}\right\}_{\varepsilon}$, with $F_{\varepsilon}$ given by (1.4), $\Gamma$-converges to a certain functional, $\mathcal{F}_{\text {hom }}$, with respect to the weak topology in $L^{p}\left(\Omega ; \mathbb{R}^{\mathbb{d}}\right)$, as $\varepsilon \rightarrow 0^{+}$. Then, in Proposition 4.6 below, we establish the integral representation for this $\Gamma$-limit as stated in Theorem 1.1.

THEOREM 4.1. Let $\Omega \subset \mathbb{R}^{\mathrm{n}}$ be an open and bounded set, let $f_{R}: \Omega \times \mathbb{R}^{\mathrm{n}} \times \mathbb{R}^{\mathrm{d}} \rightarrow$ $[0, \infty)$ be a function satisfying $(\mathrm{H} 1)-(\mathrm{H} 3)$, let $F_{\varepsilon}$ be the functional introduced in (1.4), and assume that (1.6) holds. Then, the sequence $\left\{F_{\varepsilon}\right\}_{\varepsilon} \Gamma$-converges on $\mathcal{U}_{\mathcal{A}}=\{u \in$ $\left.L^{p}\left(\Omega ; \mathbb{R}^{\mathbb{d}}\right): \mathcal{A} u=0\right\}$ as $\varepsilon \rightarrow 0^{+}$, with respect to the weak topology in $L^{p}\left(\Omega ; \mathbb{R}^{\mathrm{d}}\right)$, to the functional $\mathcal{F}_{\text {hom }}$ defined, for $u \in \mathcal{U}_{\mathcal{A}}$, by

$$
\mathcal{F}_{\text {hom }}(u):=\inf _{w \in \mathcal{W}_{\mathcal{A}}} \int_{\Omega} f_{Y^{\mathrm{m}}} f(x, y, u(x)+w(x, y)) \mathrm{d} x \mathrm{~d} y,
$$

where

$$
\begin{gathered}
\mathcal{W}_{\mathcal{A}}:=\left\{w \in L^{p}\left(\Omega ; L_{\#}^{p}\left(Y^{\mathrm{m}} ; \mathbb{R}^{\mathrm{d}}\right)\right): w \text { is }\left(\mathcal{A}, \mathcal{A}_{\boldsymbol{R}^{*}}^{y}\right)\right. \text {-free in the sense of Definition 3.13, } \\
\text { with } \left.\int_{Y^{\mathrm{m}}} w(\cdot, y) \mathrm{d} y=0\right\} .
\end{gathered}
$$

Precisely, given an arbitrary sequence $\left\{\varepsilon_{n}\right\}_{n \in \mathbb{N}} \subset \mathbb{R}^{+}$converging to 0 , the following pair of statements holds: 
1. (Г-liminf inequality). Let $\left\{u_{n}\right\}_{n \in \mathbb{N}} \subset \mathcal{U}_{\mathcal{A}}$ be a sequence such that $u_{n} \rightarrow u$ in $L^{p}\left(\Omega ; \mathbb{R}^{\mathbb{d}}\right)$ for some $u \in L^{p}\left(\Omega ; \mathbb{R}^{\mathbb{d}}\right)$. Then, $u \in \mathcal{U}_{\mathcal{A}}$ and

$$
\liminf _{n \rightarrow \infty} F_{\varepsilon_{n}}\left(u_{n}\right) \geqslant \mathcal{F}_{\text {hom }}(u) .
$$

2. (recovery sequence). For every $u \in \mathcal{U}_{\mathcal{A}}$, there exists sequence $\left\{u_{n}\right\}_{n \in \mathbb{N}} \subset \mathcal{U}_{\mathcal{A}}$ such that $u_{n} \rightarrow u$ in $L^{p}\left(\Omega ; \mathbb{R}^{\mathrm{d}}\right)$ and

$$
\limsup _{n \rightarrow \infty} F_{\varepsilon_{n}}\left(u_{n}\right) \leqslant \mathcal{F}_{\text {hom }}(u) .
$$

The proof of Theorem 4.1 is obtained as a consequence of Propositions 4.3 and 4.4 below. We begin with a lemma that will be used in the subsequent proposition, where we establish the recovery sequence property, and is a simple adaptation of [31, Proposition 3.5-(i)].

Lemma 4.2. Assume that hypotheses (H1)-(H2) hold. Let $\left\{\varepsilon_{n}\right\}_{n \in \mathbb{N}} \subset \mathbb{R}^{+}$be a sequence converging to 0 , and let $\left\{u_{n}\right\}_{n \in \mathbb{N}},\left\{w_{n}\right\}_{n \in \mathbb{N}} \subset L^{p}\left(\Omega ; \mathbb{R}^{\mathbb{d}}\right)$ be two p-equiintegrable sequences such that $\lim _{n \rightarrow \infty}\left\|u_{n}-w_{n}\right\|_{L^{p}\left(\Omega ; \mathbb{R}^{\mathrm{d}}\right)}=0$. Then,

$$
\lim _{n \rightarrow \infty} \int_{\Omega}\left[f_{R}\left(x, \frac{x}{\varepsilon_{n}}, u_{n}(x)\right) \mathrm{d} x-f_{R}\left(x, \frac{x}{\varepsilon_{n}}, w_{n}(x)\right)\right] \mathrm{d} x=0 .
$$

Proof. Fix $\tau>0$. We want to show that there exists $n_{0}=n_{0}(\tau) \in \mathbb{N}$ such that if $n \geqslant n_{0}$, then

$$
\left|\int_{\Omega}\left[f_{R}\left(x, \frac{x}{\varepsilon_{n}}, u_{n}(x)\right) \mathrm{d} x-f_{R}\left(x, \frac{x}{\varepsilon_{n}}, w_{n}(x)\right)\right] \mathrm{d} x\right| \leqslant \tau .
$$

Using the $p$-equi-integrability of $\left\{u_{n}\right\}$ and $\left\{w_{n}\right\}$, there exists $\delta=\delta(\tau)>0$ such that if $E \subset \Omega$ is a measurable set with $|E|<\delta$, then

$$
\sup _{n \in \mathbb{N}} \int_{E} C\left(2+\left|u_{n}(x)\right|^{p}+\left|w_{n}(x)\right|^{p}\right) \mathrm{d} x<\frac{\tau}{8} .
$$

Moreover, there exists $r_{\delta}>0$ such that

$$
\sup _{n \in \mathbb{N}}\left[\left|\left\{\left|u_{n}\right| \geqslant r_{\delta}\right\}\right|+\left|\left\{\left|w_{n}\right| \geqslant r_{\delta}\right\}\right|\right] \leqslant \delta .
$$

Let $\Omega_{\delta} \Subset \Omega$ be such that $\left|\Omega \backslash \Omega_{\delta}\right| \leqslant \delta$. Using the continuity assumption on $f$ and the $Y^{\mathrm{m}}$-periodicity of $f$ with respect to its second variable, we conclude that $f$ is uniformly continuous on $\bar{\Omega}_{\delta} \times \mathbb{R}^{\mathrm{m}} \times \bar{B}_{r_{\delta}}(0)$. Thus, we can find $0<\bar{\delta} \leqslant \delta$ such that, for all $x \in \Omega_{\delta}, y \in \mathbb{R}^{\mathrm{m}}$, and $\xi_{1}, \xi_{2} \in B_{r_{\delta}}(0)$ with $\left|\xi_{1}-\xi_{2}\right| \leqslant \bar{\delta}$, we have

$$
\left|f\left(x, y, \xi_{1}\right)-f\left(x, y, \xi_{2}\right)\right| \leqslant \frac{\tau}{2\left|\Omega_{\delta}\right|} .
$$

Finally, by Chebyshev's inequality, there exists $0<\tilde{\delta}<\bar{\delta}$ such that if $\|v\|_{L^{p}\left(\Omega ; \mathbb{R}^{d}\right)}<$ $\tilde{\delta}$, then

$$
|\{|v| \geqslant \bar{\delta}\}| \leqslant \delta
$$

We observe further that because $\lim _{n \rightarrow \infty}\left\|u_{n}-w_{n}\right\|_{L^{p}\left(\Omega ; \mathbb{R}^{\mathrm{d}}\right)}=0$, we can find $n_{0}=$ $n_{0}(\tau) \in \mathbb{N}$ such that $\left\|u_{n}-w_{n}\right\|_{L^{p}\left(\Omega ; \mathbb{R}^{\mathrm{d}}\right)}<\tilde{\delta}$ for all $n \geqslant n_{0}$. 
Thus, for each $n \geqslant n_{0}$ and for $A:=\left(\Omega \backslash \Omega_{\delta}\right) \cup\left\{\left|u_{n}\right| \geqslant r_{\delta}\right\} \cup\left\{\left|w_{n}\right| \geqslant r_{\delta}\right\} \cup\left\{\mid u_{n}-\right.$ $\left.w_{n} \mid \geqslant \bar{\delta}\right\}$, we conclude from (H2), (H3), and (4.2)-(4.5) that

$$
\begin{aligned}
& \left|\int_{\Omega}\left[f_{R}\left(x, \frac{x}{\varepsilon_{n}}, u_{n}(x)\right) \mathrm{d} x-f_{R}\left(x, \frac{x}{\varepsilon_{n}}, w_{n}(x)\right)\right] \mathrm{d} x\right| \\
& \quad \leqslant \int_{A} C\left(2+\left|u_{n}(x)\right|^{p}+\left|w_{n}(x)\right|^{p}\right) \mathrm{d} x \\
& \quad+\left|\int_{\Omega \backslash A}\left[f\left(x, \frac{\boldsymbol{R} x}{\varepsilon_{n}}, u_{n}(x)\right) \mathrm{d} x-f\left(x, \frac{\boldsymbol{R} x}{\varepsilon_{n}}, w_{n}(x)\right)\right] \mathrm{d} x\right| \\
& \quad \leqslant \frac{\tau}{2}+\frac{\tau}{2\left|\Omega_{\delta}\right|}|\Omega \backslash A| \leqslant \tau .
\end{aligned}
$$

The recovery sequence property in Theorem 4.1 is a simple consequence of the following proposition. We observe that this result does not require assumption (H3) to hold and is the quasi-periodic counterpart of [31, Proposition 2.7] concerning the periodic case.

Proposition 4.3. Assume that hypotheses $(\mathrm{H} 1)-(\mathrm{H} 2)$ hold, and let $\mathcal{U}_{\mathcal{A}}$ be the set introduced in (1.9). Then, for each $\delta>0, u \in \mathcal{U}_{\mathcal{A}}$, and $w \in \overline{\mathcal{W}}_{\mathcal{A}}:=\{w \in$ $L^{p}\left(\Omega ; L_{\#}^{p}\left(Y^{\mathrm{m}} ; \mathbb{R}^{\mathrm{d}}\right)\right): w$ is $\left(\mathcal{A}, \mathcal{A}_{\boldsymbol{R}^{*}}^{y}\right)$-free in the sense of Definition 3.13$\}$, there exists a sequence $\left\{u_{\varepsilon}\right\} \subset \mathcal{U}_{\mathcal{A}}$ such that $u_{\varepsilon} \rightarrow u+\bar{w}_{0}$ weakly in $L^{p}\left(\Omega ; \mathbb{R}^{\mathrm{d}}\right)$ as $\varepsilon \rightarrow 0^{+}$and, for all $\kappa \in \mathbb{N}$,

$$
\lim _{\varepsilon \rightarrow 0^{+}} \int_{\Omega} f_{R}\left(x, \frac{x}{\varepsilon}, u_{\varepsilon}(x)\right) \mathrm{d} x \leqslant \int_{\Omega} f_{Y^{\mathrm{m}}} f(x, \kappa y, u(x)+w(x, y)) \mathrm{d} y \mathrm{~d} x+\delta,
$$

where, recalling Definition 3.13 , we have $\bar{w}_{0}:=f_{Y^{\mathrm{m}}} w(\cdot, y) \mathrm{d} y$.

Proof. Fix $\delta>0, u \in \mathcal{U}_{\mathcal{A}}$, and $w \in \overline{\mathcal{W}}_{\mathcal{A}}$. We will proceed in two steps, first assuming extra regularity on $w$, and then treating the general case.

Step 1. Recalling the decomposition $w=\bar{w}_{0}+\bar{w}_{1}$ introduced in Definition 3.13, assume that $\bar{w}_{1} \in C^{1}\left(\bar{\Omega} ; C_{\#}^{1}\left(Y^{\mathrm{m}} ; \mathbb{R}^{\mathrm{d}}\right)\right)$.

For $\kappa \in \mathbb{N}$ and $(x, y) \in \Omega \times Y^{\mathrm{m}}$, define

$$
\psi(x, y):=f(x, \kappa y, u(x)+w(x, y))=f\left(x, \kappa y, u(x)+\bar{w}_{0}(x)+\bar{w}_{1}(x, y)\right) .
$$

Using (H1), (H2), the continuity of $f$, and the regularity of $\bar{w}_{1}$, we conclude that $\psi \in L^{1}\left(\Omega ; C_{\#}\left(Y^{\mathrm{m}}\right)\right)$. Then, by Proposition 3.7 , we have

$$
\lim _{\varepsilon \rightarrow 0^{+}} \int_{\Omega} \psi\left(x, \frac{\boldsymbol{R} x}{\varepsilon}\right) \mathrm{d} x=\int_{\Omega} f_{Y^{\mathrm{m}}} \psi(x, y) \mathrm{d} x \mathrm{~d} y,
$$

i.e.,

$$
\lim _{\varepsilon \rightarrow 0^{+}} \int_{\Omega} f_{R}\left(x, \frac{x}{\varepsilon}, w_{\varepsilon}(x)\right) \mathrm{d} x=\int_{\Omega} f_{Y^{\mathrm{m}}} f(x, \kappa y, u(x)+w(x, y)) \mathrm{d} y \mathrm{~d} x,
$$

where, for $x \in \Omega$,

$$
w_{\varepsilon}(x):=u(x)+\bar{w}_{0}(x)+\bar{w}_{1}\left(x, \frac{\boldsymbol{R} x}{\varepsilon}\right) .
$$

Arguing as in Step 1 of the proof of Proposition 3.15 with $\bar{u}_{1}$ replaced by $\bar{w}_{1}$ in (3.11), and using the fact that $\mathcal{A} u+\mathcal{A} \bar{w}_{0}=0$ by the definition of $\mathcal{U}_{\mathcal{A}}$ and $\overline{\mathcal{W}}_{\mathcal{A}}$, we 
conclude that (see (3.12)-(3.13))

$$
\begin{aligned}
& \left\{w_{\varepsilon}\right\}_{\varepsilon} \text { is a } p \text {-equi-integrable sequence in } L^{p}\left(\Omega ; \mathbb{R}^{\mathbb{d}}\right), \\
& w_{\varepsilon} \rightarrow u+\bar{w}_{0} \text { weakly in } L^{p}\left(\Omega ; \mathbb{R}^{\mathbb{d}}\right), \\
& \mathcal{A} w_{\varepsilon} \rightarrow 0 \text { in } W^{-1, p}\left(\Omega ; \mathbb{R}^{\mathbb{1}}\right) .
\end{aligned}
$$

Then, by Lemma 2.3 , we can find a sequence $\left\{u_{\varepsilon}\right\}_{\varepsilon} \subset L^{p}\left(\Omega ; \mathbb{R}^{d}\right)$ such that

$$
\begin{aligned}
& \left\{u_{\varepsilon}\right\}_{\varepsilon} \text { is } p \text {-equi-integrable, } \\
& \mathcal{A} u_{\varepsilon}=0 \text { in } L^{p}\left(\Omega ; \mathbb{R}^{\mathbb{1}}\right), \\
& u_{\varepsilon}-w_{\varepsilon} \rightarrow 0 \text { in } L^{p}\left(\Omega ; \mathbb{R}^{\mathbb{d}}\right) .
\end{aligned}
$$

In particular, $u_{\varepsilon} \rightarrow u+\bar{w}_{0}$ weakly in $L^{p}\left(\Omega ; \mathbb{R}^{\mathrm{d}}\right)$. Moreover, by Lemma 4.2 , we have

$$
\lim _{\varepsilon \rightarrow 0^{+}} \int_{\Omega} f_{R}\left(x, \frac{x}{\varepsilon}, u_{\varepsilon}(x)\right) \mathrm{d} x=\lim _{\varepsilon \rightarrow 0^{+}} \int_{\Omega} f_{R}\left(x, \frac{x}{\varepsilon}, w_{\varepsilon}(x)\right) \mathrm{d} x,
$$

which, together with (4.7), concludes Step 1.

Step 2. We treat the general case.

Fix $j \in \mathbb{N}$. Arguing as in Step 1 of the proof of Proposition 3.15 with $\bar{u}_{1}$ replaced by $\bar{w}_{1}$, we can find $\tilde{w}_{j} \in C^{1}\left(\bar{\Omega} ; C_{\#}^{1}\left(Y^{\mathrm{m}} ; \mathbb{R}^{\mathrm{d}}\right)\right)$ such that $\bar{w}_{0}+\tilde{w}_{j} \in \overline{\mathcal{W}}_{\mathcal{A}}$ and $\| \tilde{w}_{j}-$ $\bar{w}_{1} \|_{L^{p}\left(\Omega ; L_{\#}^{p}\left(Y^{\mathrm{m}} ; \mathbb{R}^{\mathrm{d}}\right)\right)} \leqslant \frac{1}{j}$. Then, extracting a subsequence of $\left\{\tilde{w}_{j}\right\}_{j \in \mathbb{N}}$ if necessary, the Vitali-Lebesgue theorem and (H1)-(H2) yield

$$
\begin{gathered}
\lim _{j \rightarrow \infty} \int_{\Omega} f_{Y^{\mathrm{m}}} f\left(x, \kappa y, u(x)+\bar{w}_{0}(x)+\tilde{w}_{j}(x, y)\right) \mathrm{d} y \mathrm{~d} x \\
=\int_{\Omega} f_{Y^{\mathrm{m}}} f\left(x, \kappa y, u(x)+\bar{w}_{0}(x)+\bar{w}_{1}(x, y)\right) \mathrm{d} y \mathrm{~d} x .
\end{gathered}
$$

Hence, we can find $j_{\delta} \in \mathbb{N}$ such that

$$
\begin{aligned}
& \int_{\Omega} f_{Y^{\mathrm{m}}} f\left(x, \kappa y, u(x)+\bar{w}_{0}(x)+\tilde{w}_{j \delta}(x, y)\right) \mathrm{d} y \mathrm{~d} x \\
& \quad \leqslant \int_{\Omega} f_{Y^{\mathrm{m}}} f\left(x, \kappa y, u(x)+\bar{w}_{0}(x)+\bar{w}_{1}(x, y)\right) \mathrm{d} y \mathrm{~d} x+\delta .
\end{aligned}
$$

To conclude, we invoke Step 1 to find a sequence $\left\{u_{\varepsilon}\right\} \subset \mathcal{U}_{\mathcal{A}}$ that depends on $\delta$, $u$, and $w$, such that $u_{\varepsilon} \rightarrow u+\bar{w}_{0}$ weakly in $L^{p}\left(\Omega ; \mathbb{R}^{\mathbb{d}}\right)$ as $\varepsilon \rightarrow 0^{+}$and, for all $\kappa \in \mathbb{N}$,

$$
\lim _{\varepsilon \rightarrow 0^{+}} \int_{\Omega} f_{R}\left(x, \frac{x}{\varepsilon}, u_{\varepsilon}(x)\right) \mathrm{d} x=\int_{\Omega} f_{Y^{\mathrm{m}}} f\left(x, \kappa y, u(x)+\bar{w}_{0}(x)+\tilde{w}_{j_{\delta}}(x, y)\right) \mathrm{d} y \mathrm{~d} x .
$$

Next, we establish the $\Gamma$-liminf inequality property stated in Theorem 4.1.

Proposition 4.4. Let $\left\{\varepsilon_{n}\right\}_{n \in \mathbb{N}} \subset \mathbb{R}^{+}$be a sequence converging to 0 , and let $\left\{u_{n}\right\}_{n \in \mathbb{N}} \subset \mathcal{U}_{\mathcal{A}}$ be a sequence such that $u_{n} \rightarrow u$ in $L^{p}\left(\Omega ; \mathbb{R}^{\mathrm{d}}\right)$ for some $u \in L^{p}\left(\Omega ; \mathbb{R}^{\mathrm{d}}\right)$. Then, under the assumptions of Theorem 4.1, we have $u \in \mathcal{U}_{\mathcal{A}}$ and

$$
\liminf _{n \rightarrow \infty} F_{\varepsilon_{n}}\left(u_{n}\right) \geqslant \mathcal{F}_{\text {hom }}(u) .
$$

Copyright $@$ by SIAM. Unauthorized reproduction of this article is prohibited. 
Proof. The condition $u \in \mathcal{U}_{\mathcal{A}}$ follows from the fact that $u_{n} \in \mathcal{U}_{\mathcal{A}}$ for all $n \in$ $\mathbb{N}$ together with the convergence $u_{n} \rightarrow u$ in $L^{p}\left(\Omega ; \mathbb{R}^{\mathbb{d}}\right)$. Moreover, by Propositions 3.14 and 3.4 and the uniqueness of $\boldsymbol{R}$-two-scale limits (see Remark 3.2), we have $u_{n} \stackrel{\boldsymbol{R} \text {-2sc }}{\longrightarrow} v$ for a vector-field $v$ that is $\left(\mathcal{A}, \mathcal{A}_{\boldsymbol{R}^{*}}^{y}\right)$-free in the sense of Definition 3.13 , with $\int_{Y^{\mathrm{m}}} v(\cdot, y) \mathrm{d} y=u(\cdot)$. In particular, we have the decomposition

$$
v=u+v_{1}, \quad v_{1} \in L^{p}\left(\Omega ; L_{\#}^{p}\left(Y^{\mathrm{m}} ; \mathbb{R}^{\mathrm{d}}\right)\right), \quad \mathcal{A}_{\boldsymbol{R}^{*}}^{y} v_{1}=0, \quad \int_{Y^{\mathrm{m}}} v_{1}(\cdot, y) \mathrm{d} y=0 .
$$

Let $\left\{\psi_{j}\right\}_{j \in \mathbb{N}} \subset C_{c}\left(\Omega ; C_{\#}\left(Y^{\mathrm{m}} ; \mathbb{R}^{\mathbb{1}}\right)\right)$ be a sequence converging to $v$ in $L^{p}(\Omega \times$ $\left.Y^{\mathrm{m}} ; \mathbb{R}^{\mathrm{d}}\right)$ and pointwise in $\Omega \times Y^{\mathrm{m}}$. By (H3), we have, for all $n, j \in \mathbb{N}$,

$$
\begin{aligned}
f\left(x, \frac{\boldsymbol{R} x}{\varepsilon_{n}}, u_{n}(x)\right) \geqslant & f\left(x, \frac{\boldsymbol{R} x}{\varepsilon_{n}}, \psi_{j}\left(x, \frac{\boldsymbol{R} x}{\varepsilon_{n}}\right)\right) \\
& +\frac{\partial f}{\partial \xi}\left(x, \frac{\boldsymbol{R} x}{\varepsilon}, \psi_{j}\left(x, \frac{\boldsymbol{R} x}{\varepsilon}\right)\right) \cdot\left(u_{n}(x)-\psi_{j}\left(x, \frac{\boldsymbol{R} x}{\varepsilon}\right)\right) .
\end{aligned}
$$

Integrating this estimate over $\Omega$ and passing to the limit as $n \rightarrow \infty$, we invoke Proposition 3.7 and $(\mathrm{H} 2)-(\mathrm{H} 3)$ to infer that

$$
\begin{aligned}
& \liminf _{n \rightarrow \infty} F_{\varepsilon_{n}}\left(u_{n}\right)=\liminf _{n \rightarrow \infty} \int_{\Omega} f\left(x, \frac{\boldsymbol{R} x}{\varepsilon_{n}}, u_{n}(x)\right) \mathrm{d} x \\
& \geqslant \int_{\Omega} f_{Y^{\mathrm{m}}} f\left(x, y, \psi_{j}(x, y)\right) \mathrm{d} x \mathrm{~d} y+\int_{\Omega} f_{Y^{\mathrm{m}}} \frac{\partial f}{\partial \xi}\left(x, y, \psi_{j}(x, y)\right) \cdot\left(v(x, y)-\psi_{j}(x, y)\right) \mathrm{d} x \mathrm{~d} y
\end{aligned}
$$

for all $j \in \mathbb{N}$. Letting $j \rightarrow \infty$ in this inequality, Fatou's lemma and (H1) yield

$$
\begin{aligned}
\liminf _{n \rightarrow \infty} F_{\varepsilon_{n}}\left(u_{n}\right) & \geqslant \int_{\Omega} f_{Y^{\mathrm{m}}} f(x, y, v(x, y)) \mathrm{d} y \mathrm{~d} x=\int_{\Omega} f_{Y^{\mathrm{m}}} f\left(x, y, u(x)+v_{1}(x, y)\right) \mathrm{d} y \mathrm{~d} x \\
& \geqslant \inf _{w \in \mathcal{W}_{\mathcal{A}}} \int_{\Omega} f_{Y^{\mathrm{m}}} f(x, y, u(x)+w(x, y)) \mathrm{d} x \mathrm{~d} y=\mathcal{F}_{\mathrm{hom}}(u) .
\end{aligned}
$$

Proof of Theorem 4.1. Proving that both the $\Gamma$-liminf inequality and the recovery sequence properties in Theorem 4.1 hold is equivalent to proving that (see [22]) for all $u \in \mathcal{U}_{\mathcal{A}}$, we have

$$
\mathcal{F}_{\text {hom }}(u)=\Gamma-\liminf _{n \rightarrow \infty} F_{\varepsilon_{n}}(u)=\Gamma-\limsup _{n \rightarrow \infty} F_{\varepsilon_{n}}(u),
$$

where $\left\{\varepsilon_{n}\right\}_{n \in \mathbb{N}} \subset \mathbb{R}^{+}$is an arbitrary sequence converging to 0 and

$$
\begin{aligned}
& \Gamma-\liminf _{n \rightarrow \infty} F_{\varepsilon_{n}}(u) \\
& :=\inf \left\{\liminf _{n \rightarrow \infty} F_{\varepsilon_{n}}\left(u_{n}\right): u_{n} \rightarrow u \text { in } L^{p}\left(\Omega ; \mathbb{R}^{\mathrm{d}}\right) \text { as } n \rightarrow \infty, \AA u_{n}=0 \text { for all } n \in \mathbb{N}\right\}, \\
& \Gamma-\limsup _{n \rightarrow \infty} F_{\varepsilon_{n}}(u) \\
& :=\inf \left\{\limsup _{n \rightarrow \infty} F_{\varepsilon_{n}}\left(u_{n}\right): u_{n} \rightarrow u \text { in } L^{p}\left(\Omega ; \mathbb{R}^{\mathrm{d}}\right) \text { as } n \rightarrow \infty, \AA u_{n}=0 \text { for all } n \in \mathbb{N}\right\} .
\end{aligned}
$$

Copyright (c) by SIAM. Unauthorized reproduction of this article is prohibited. 
Taking the infimum over all admissible sequences on (4.8), we conclude from Proposition 4.4 that

$$
\Gamma-\liminf _{n \rightarrow \infty} F_{\varepsilon_{n}}(u) \geqslant \mathcal{F}_{\text {hom }}(u) .
$$

On the other hand, Proposition 4.3 with $\kappa=1$ and $\bar{w}_{0}=0$ yields

$$
\Gamma-\limsup _{n \rightarrow \infty} F_{\varepsilon_{n}}(u) \leqslant \int_{\Omega} f_{Y^{\mathrm{m}}} f\left(x, \kappa y, u(x)+\bar{w}_{1}(x, y)\right) \mathrm{d} y \mathrm{~d} x+\delta
$$

for all $\delta>0$ and $\bar{w}_{1} \in \mathcal{W}_{\mathcal{A}}$. Hence, taking the infimum over $\bar{w}_{1} \in \mathcal{W}_{\mathcal{A}}$, and then letting $\delta \rightarrow 0$, we get

$$
\Gamma-\limsup _{n \rightarrow \infty} F_{\varepsilon_{n}}(u) \leqslant \mathcal{F}_{\text {hom }}(u) .
$$

Because $\Gamma$ - $\liminf \operatorname{in}_{n \rightarrow \infty} F_{\varepsilon_{n}}(u) \leqslant \Gamma-\lim \sup _{n \rightarrow \infty} F_{\varepsilon_{n}}(u)$, we obtain (4.9) from (4.10) and (4.11).

Next, we establish an integral representation for the functional $\mathcal{F}_{\text {hom }}$ introduced in Theorem 4.1. This integral representation is the quasi-periodic counterpart of [31, Corollary 3.2] concerning the periodic case, and its proof uses the following measurable selection criterion, proved in [31, Lemma 3.10] (also see [18]).

Lemma 4.5. Let $Z$ be a separable metric space, let $T$ be a measurable space, and let $\Upsilon: T \rightarrow 2^{Z}$ be a multivalued function such that (i) for every $t \in T, \Upsilon(t) \subset Z$ is nonempty and open; and (ii) for every $z \in Z,\{t \in T: z \in \Upsilon(t)\} \subset T$ is measurable. Then, $\Upsilon$ admits a measurable selection; that is, there exists a measurable function, $v: T \rightarrow Z$, such that $v(t) \in \Upsilon(t)$ for all $t \in T$.

Proposition 4.6. Under the assumptions of Theorem 4.1, for all $u \in \mathcal{U}_{\mathcal{A}}$, we have

$$
\mathcal{F}_{\text {hom }}(u)=\int_{\Omega} f_{\text {hom }}(x, u(x)) \mathrm{d} x,
$$

where

$$
f_{\text {hom }}(x, \xi)=\inf _{v \in \mathcal{V}_{\mathcal{A}}} f_{Y^{\mathrm{m}}} f(x, y, \xi+v(y)) \mathrm{d} y
$$

with $\mathcal{V}_{\mathcal{A}}$ given by $(1.10)$

Proof. Let $u \in \mathcal{U}_{\mathcal{A}}$. Note that, by (H2), we have

$$
0 \leqslant f_{\text {hom }}(x, \xi) \leqslant C\left(1+|\xi|^{p}\right)
$$

for all $(x, \xi) \in \Omega \times \mathbb{R}^{\mathrm{d}}$. Moreover,

$$
x \in \Omega \mapsto f_{\text {hom }}(x, u(x))
$$

is a measurable map. In fact, let $V_{\mathcal{A}}$ be a countable and dense subset of $\mathcal{V}_{\mathcal{A}}$ with respect to the (strong) topology of $L_{\#}^{p}\left(Y^{\mathrm{m}} ; \mathbb{R}^{\mathrm{d}}\right)$. We observe that such a set $V_{\mathcal{A}}$ exists because $\mathcal{V}_{\mathcal{A}}$ is a subset of the separable metric space $L_{\#}^{p}\left(Y^{\mathrm{m}} ; \mathbb{R}^{\mathrm{d}}\right)$. Then, the continuity of $f$ (see (H1)), the Vitali-Lebesgue theorem, and (H2) yield

$$
\inf _{v \in \mathcal{V}_{\mathcal{A}}} f_{Y^{\mathrm{m}}} f(x, y, u(x)+v(y)) \mathrm{d} y=\inf _{v \in V_{\mathcal{A}}} f_{Y^{\mathrm{m}}} f(x, y, u(x)+v(y)) \mathrm{d} y,
$$

Copyright $@$ by SIAM. Unauthorized reproduction of this article is prohibited. 
from which we conclude the measurability of the map in (4.14).

Fix $w \in \mathcal{W}_{\mathcal{A}}$. For a.e. $x \in \Omega$, we have $w(x, \cdot) \in \mathcal{V}_{\mathcal{A}}$; hence, for a.e. $x \in \Omega$,

$$
\inf _{v \in \mathcal{V}_{\mathcal{A}}} f_{Y^{\mathrm{m}}} f(x, y, u(x)+v(y)) \mathrm{d} y \leqslant f_{Y^{\mathrm{m}}} f(x, y, u(x)+w(x, y)) \mathrm{d} y .
$$

Integrating this estimate over $\Omega$, and then taking the infimum over $w \in \mathcal{W}_{\mathcal{A}}$, we conclude that

$$
\int_{\Omega} f_{\text {hom }}(x, u(x)) \mathrm{d} x \leqslant \mathcal{F}_{\text {hom }}(u) .
$$

To prove the converse inequality, we first observe that, by (4.13), we may assume that

$$
f_{\text {hom }}(x, u(x)) \in \mathbb{R} \text { for all } x \in \Omega
$$

without loss of generality. Fix $\delta>0$, and consider the multivalued function $\Upsilon_{\delta}: \Omega \rightarrow$ $2^{L_{\#}^{p}\left(Y^{\mathrm{m}} ; \mathbb{R}^{\mathrm{d}}\right)}$ defined, for $x \in \Omega$, by

$$
\Upsilon_{\delta}(x):=\left\{v \in \mathcal{V}_{\mathcal{A}}: f_{Y^{\mathrm{m}}} f(x, y, u(x)+v(y)) \mathrm{d} y<f_{\text {hom }}(x, u(x))+\delta\right\} .
$$

Also, let $\bar{\delta} \in(0, \delta)$ be such that

$$
\int_{E} C\left(1+|u(x)|^{p}\right) \mathrm{d} x<\delta
$$

whenever $E \subset \Omega$ is a measurable set with $\mathcal{L}^{\mathrm{n}}(E)<\bar{\delta}$, where $C$ is given by (H2).

By (4.15), we have $\Upsilon_{\delta}(x) \neq \emptyset$ for all $x \in \Omega$. Furthermore, arguing as above, using the continuity of $f$, the Vitali-Lebesgue theorem, and (H2), it can be checked that for each $x \in \Omega, L_{\#}^{p}\left(Y^{\mathrm{m}} ; \mathbb{R}^{\mathrm{d}}\right) \backslash \Upsilon_{\delta}(x)$ is a closed subset of $L_{\#}^{p}\left(Y^{\mathrm{m}} ; \mathbb{R}^{\mathrm{d}}\right)$. On the other hand, recalling the measurability of the map in (4.14), we have that

$$
x \mapsto h(x):=f_{Y^{\mathrm{m}}} f(x, y, u(x)+v(y)) \mathrm{d} y-f_{\mathrm{hom}}(x, u(x))-\delta
$$

defines a measurable map for each $v \in L_{\#}^{p}\left(Y^{\mathrm{m}} ; \mathbb{R}^{\mathrm{d}}\right)$. Thus, $\left\{x \in \Omega: v \in \Upsilon_{\delta}(x)\right\}=$ $h^{-1}((-\infty, 0))$ is a measurable set for each $v \in L_{\#}^{p}\left(Y^{\mathrm{m}} ; \mathbb{R}^{\mathrm{d}}\right)$. Consequently, by Lemma 4.5, there exists a measurable selection, $w_{\delta}: \Omega \rightarrow L_{\#}^{p}\left(Y^{\mathrm{m}} ; \mathbb{R}^{\mathrm{d}}\right)$, of $\Upsilon_{\delta}$. Moreover, by Lusin's theorem, $w_{\delta} \in L^{p}\left(\Omega_{\delta} ; L_{\#}^{p}\left(Y^{\mathrm{m}} ; \mathbb{R}^{\mathrm{d}}\right)\right)$ for a suitable measurable set $\Omega_{\delta}$ such that $\mathcal{L}^{\mathrm{m}}\left(\Omega \backslash \Omega_{\delta}\right)<\bar{\delta}$.

Finally, we define $\bar{w}_{\delta} \in \mathcal{W}_{\mathcal{A}}$ by setting $\bar{w}_{\delta}(x):=w_{\delta}(x)$ if $x \in \Omega_{\delta}$, and $\bar{w}_{\delta}(x):=0$ if $x \in \Omega \backslash \Omega_{\delta}$. Then, using the definition of $\mathcal{F}_{\text {hom }}(u)$, (H2), (4.16), and (4.13), we get

$$
\begin{aligned}
\mathcal{F}_{\text {hom }}(u) & \leqslant \int_{\Omega} f_{Y^{\mathrm{m}}} f\left(x, y, u(x)+\bar{w}_{\delta}(x, y)\right) \mathrm{d} x \mathrm{~d} y \\
& \leqslant \delta+\int_{\Omega_{\delta}} f_{Y^{\mathrm{m}}} f\left(x, y, u(x)+w_{\delta}(x, y)\right) \mathrm{d} x \mathrm{~d} y \\
& \leqslant \delta\left(1+\mathcal{L}^{\mathrm{n}}(\Omega)\right)+\int_{\Omega} f_{\text {hom }}(x, u(x)) \mathrm{d} x,
\end{aligned}
$$

from which we conclude the desired inequality by letting $\delta \rightarrow 0$.

Copyright (c) by SIAM. Unauthorized reproduction of this article is prohibited. 
Finally, we prove Theorem 1.1.

Proof of Theorem 1.1. Theorem 1.1 is an immediate consequence of Theorem 4.1 (also see (4.9)) and Proposition 4.6.

5. The curl case. In this section, we prove Theorem 1.4 that provides an equivalent alternative characterization for the $\boldsymbol{R}$-two-scale limit of bounded sequences in $W^{1, p}$, corresponding to $\mathcal{A}=$ curl in Theorem 1.3. In this case, by Proposition 3.9 and extracting a subsequence if necessary, we have $u_{\varepsilon} \stackrel{\boldsymbol{R}-2 s c}{\longrightarrow} u_{0}$ and $\nabla u_{\varepsilon} \stackrel{\boldsymbol{R}-2 s c}{\longrightarrow} U_{0}$ for some $u_{0} \in L^{p}\left(\Omega \times Y^{\mathrm{m}}\right)$ and $U_{0} \in L^{p}\left(\Omega \times Y^{\mathrm{m}} ; \mathbb{R}^{\mathrm{m}}\right)$. Next, to study the relationship between $u_{0}$ and $U_{0}$, we use Fourier analysis. As we mentioned before, this is the approach adopted in [8]; however, the arguments in [8] hinge on the Parseval and Plancherel identities, which are valid in $L^{2}(\Omega)$ only. Instead, our main tool here relies on the following theorem, which may be found in [34]. For simplicity, we take

$$
Y^{\mathrm{m}}=[0,1)^{\mathrm{m}},
$$

and we use the Einstein convention on repeated indices.

Theorem 5.1. Let $w \in L_{\#}^{p}\left(Y^{\mathrm{m}}\right)$, and define $e^{1}$

$$
w_{N}(y):=\sum_{\substack{k \in \mathbb{Z}^{\mathrm{m}} \\|k| \infty \leqslant N}} \hat{w}_{k} e^{2 \pi i k \cdot y}, y \in Y^{\mathrm{m}}, N \in \mathbb{N},
$$

where $\hat{w}_{k}:=\int_{Y^{\mathrm{m}}} w(y) e^{-2 \pi i k \cdot y} \mathrm{~d} y, k \in \mathbb{Z}^{\mathrm{m}}$, are the Fourier coefficients of $w$. Then,

$$
\begin{aligned}
& \left\|\sup _{N \in \mathbb{N}}\left|w_{N}\right|\right\|_{L^{p}\left(Y^{\mathrm{m}}\right)} \leqslant C_{p, m}\|w\|_{L^{p}\left(Y^{\mathrm{m}}\right)}, \\
& \lim _{N \rightarrow \infty}\left\|w_{N}-w\right\|_{L^{p}\left(Y^{\mathrm{m}}\right)}=0, \\
& \lim _{N \rightarrow \infty} w_{N}(y)=w(y) \text { for a.e. } y \in Y^{\mathrm{m}},
\end{aligned}
$$

where $C_{p, m}$ is a positive constant depending on $p$ and $m$ only. Moreover, for all $k \in \mathbb{Z}^{\mathrm{m}}$,

$$
\left|\hat{w}_{k}\right|^{p} \leqslant\|w\|_{L^{p}\left(Y^{\mathrm{m}}\right)}^{p} .
$$

Proof. The proof of (5.1) may be found in [34, Thms. 4.1.8 and 4.3.16] (see also [34, Def. 3.2.3]).

To prove (5.2), we use Jensen's inequality and the equality $\left|e^{-2 \pi i k \cdot y}\right|=1, k \in \mathbb{Z}^{\mathrm{m}}$, to obtain

$$
\left|\hat{w}_{k}\right|^{p} \leqslant \int_{Y^{\mathrm{m}}}\left|w(y) e^{-2 \pi i k \cdot y}\right|^{p} \mathrm{~d} y=\|w\|_{L^{p}\left(Y^{\mathrm{m}}\right)}^{p} .
$$

Remark 5.2. Let $w \in L^{p}\left(\Omega ; L_{\#}^{p}\left(Y^{\mathrm{m}}\right)\right)$, and define

$$
v_{N}(x):=\int_{Y^{\mathrm{m}}}\left|w_{N}(x, y)-w(x, y)\right|^{p} \mathrm{~d} y, \quad x \in \Omega, \quad N \in \mathbb{N},
$$

where $w_{N}(x, y):=\sum_{\substack{k \in \mathbb{Z}^{\mathrm{m}} \\|k| \infty \leqslant N \leqslant}} \hat{w}_{k}(x) e^{2 \pi i k \cdot y}$ with $\hat{w}_{k}(x):=\int_{Y^{\mathrm{m}}} w(x, y) e^{-2 \pi i k \cdot y} \mathrm{~d} y, k \in$ $\mathbb{Z}^{\mathrm{m}}$. By (5.1), for a.e.- $x \in \Omega$, we have

$$
\sup _{N \in \mathbb{N}}\left|v_{N}(\cdot)\right| \leqslant \tilde{C}_{p, m} \int_{Y^{\mathrm{m}}}|w(\cdot, y)|^{p} \mathrm{~d} y \in L^{1}(\Omega) \text { and } \lim _{N \rightarrow \infty} v_{N}(x)=0 .
$$

\footnotetext{
${ }^{1}$ In the literature, $w_{N}$ are called the square partial sums of the Fourier series of $w$.
} 
Thus, by the Lebesgue dominated convergence theorem, it follows that $w_{N} \rightarrow w$ in $L^{p}\left(\Omega \times Y^{\mathrm{m}}\right)$ as $N \rightarrow \infty$.

Next, we study some properties of the space $\mathcal{G}_{\boldsymbol{R}}^{p}$ introduced in (1.12) that will be useful in what follows. We first observe that if $p=2$, then it can be checked that

$\mathcal{G}_{\boldsymbol{R}}^{2}=\left\{w \in L_{\#}^{2}\left(Y^{\mathrm{m}} ; \mathbb{R}^{\mathrm{m}}\right): w(y)=\sum_{k \in \mathbb{Z}^{\mathrm{m}} \backslash\{0\}} \lambda_{k} \boldsymbol{R}^{*} k e^{2 \pi i k \cdot y}\right.$ for some $\left.\left\{\lambda_{k}\right\}_{k \in \mathbb{Z}^{\mathrm{m}} \backslash\{0\}} \subset \mathbb{C}\right\}$,

and we recover the space introduced in [8].

Lemma 5.3. Assume that $\boldsymbol{R}$ satisfies (1.2). Then, the vector space $\mathcal{G}_{\boldsymbol{R}}^{p}$ introduced in (1.12) is a closed subspace of $L_{\#}^{p}\left(Y^{\mathrm{m}} ; \mathbb{R}^{\mathrm{n}}\right)$.

Proof. Let $\left\{w_{j}\right\}_{j \in \mathbb{N}} \subset \mathcal{G}_{\boldsymbol{R}}^{p}$ and $w \in L_{\#}^{p}\left(Y^{\mathrm{m}} ; \mathbb{R}^{\mathrm{n}}\right)$ be such that $\lim _{j \rightarrow \infty} \| w_{j}-$ $w \|_{L^{p}\left(Y^{\mathrm{m}} ; \mathbb{R}^{\mathrm{n}}\right)}=0$. We want to show that $w \in \mathcal{G}_{\boldsymbol{R}}^{p}$.

For each $j \in \mathbb{N}$, let $\hat{w}_{k}^{j}$ and $\hat{w}_{k}$ denote the Fourier coefficients of $w_{j}$ and $w$, respectively. By (5.2), we have $\lim _{j \rightarrow \infty}\left|\hat{w}_{k}^{j}-\hat{w}_{k}\right|=0$.

On the other hand, by definition of $\mathcal{G}_{\boldsymbol{R}}^{p}$, for each $k \in \mathbb{Z}^{\mathrm{m}}$ and $j \in \mathbb{N}$, there exists $\lambda_{k}^{j} \in \mathbb{C}$ such that $\hat{w}_{k}^{j}=\lambda_{k}^{j} \boldsymbol{R}^{*} k$ and $\lambda_{0}^{j}=0$. In particular, $\hat{w}_{0}=0$. Fix $k_{0} \in \mathbb{Z}^{\mathrm{m}} \backslash\{0\}$; by (5.2), for all $j, j^{\prime} \in \mathbb{N}$, we have

$$
\left|\lambda_{k_{0}}^{j}-\lambda_{k_{0}}^{j^{\prime}}\right|\left|\boldsymbol{R}^{*} k_{0}\right|=\left|\left(\lambda_{k_{0}}^{j}-\lambda_{k_{0}}^{j^{\prime}}\right) \boldsymbol{R}^{*} k_{0}\right|=\left|\hat{w}_{k_{0}}^{j}-\hat{w}_{k_{0}}^{j^{\prime}}\right| \leqslant\left\|w_{j}-w_{j^{\prime}}\right\|_{L^{p}\left(Y^{\mathrm{m}}: \mathbb{R}^{\mathrm{m}}\right)} .
$$

Because $\boldsymbol{R}^{*} k_{0} \neq 0$ by (1.2), we conclude that $\left\{\lambda_{k_{0}}^{j}\right\}_{j \in \mathbb{N}}$ is a Cauchy sequence in $\mathbb{C}$. Thus, there exists $\lambda_{k_{0}} \in \mathbb{C}$ such that $\lim _{j \rightarrow \infty}\left|\lambda_{k_{0}}^{j}-\lambda_{k_{0}}\right|=0$. Consequently, passing the equality $\hat{w}_{k_{0}}^{j}=\lambda_{k_{0}}^{j} \boldsymbol{R}^{*} k_{0}$ to the limit as $j \rightarrow \infty$, we obtain $\hat{w}_{k_{0}}=\lambda_{k_{0}} \boldsymbol{R}^{*} k_{0}$.

Lemma 5.4. Assume that $\boldsymbol{R}$ satisfies (1.2), and let $w_{0} \in L_{\#}^{p}\left(Y^{\mathrm{m}} ; \mathbb{R}^{\mathrm{n}}\right)$ be such that

$$
\int_{Y^{\mathrm{m}}} w_{0}(y) \cdot \psi(y) \mathrm{d} y=0
$$

for all $\psi \in C_{\#}^{\infty}\left(Y^{\mathrm{m}} ; \mathbb{R}^{\mathrm{m}}\right)$ with $\frac{\partial \psi_{l}}{\partial y_{\tau}} \boldsymbol{R}_{\tau l}=0$ in $Y^{\mathrm{m}}$, where $l \in\{1, \ldots, \mathrm{m}\}$ and $\tau \in$ $\{1, \ldots, \mathrm{m}\}$. Then, $w_{0} \in \mathcal{G}_{\boldsymbol{R}}^{p}$.

Proof. By contradiction, assume that $w_{0} \notin \mathcal{G}_{\boldsymbol{R}}^{p}$. Then, using Lemma 5.3, together with (a corollary to) the Hahn-Banach theorem (see, for instance, [14, Cor. I.8]), there exists $v \in L_{\#}^{p^{\prime}}\left(Y^{\mathrm{m}} ; \mathbb{R}^{\mathrm{m}}\right)$ such that

$$
\int_{Y^{\mathrm{m}}} v(y) \cdot w(y) \mathrm{d} y=0
$$

for all $w \in \mathcal{G}_{\boldsymbol{R}}^{p}$, and

$$
\int_{Y^{\mathrm{m}}} v(y) \cdot w_{0}(y) \mathrm{d} y \neq 0
$$

We claim that $\frac{\partial v_{l}}{\partial y_{\tau}} \boldsymbol{R}_{\tau l}=0$ in the sense of distributions. In fact, let $\phi \in C_{\#}^{\infty}\left(Y^{\mathrm{m}}\right)$, and set $w:=\boldsymbol{R}^{*} \nabla_{y} \phi$. Then, $w \in L_{\#}^{p}\left(Y^{\mathrm{m}} ; \mathbb{R}^{\mathrm{m}}\right), \hat{w}_{0}=0$, and $\hat{w}_{k}=2 \pi i \hat{\phi}_{k} \boldsymbol{R}^{*} k$ for all $k \in \mathbb{Z}^{\mathrm{m}} \backslash\{0\}$. Thus, $w \in \mathcal{G}_{\boldsymbol{R}}^{p}$ and so, by (5.4),

$$
0=\int_{Y^{\mathrm{m}}} v(y) \cdot w(y) \mathrm{d} y=\int_{Y^{\mathrm{m}}} \boldsymbol{R} v(y) \cdot \nabla_{y} \phi(y) \mathrm{d} y,
$$

Copyright (c) by SIAM. Unauthorized reproduction of this article is prohibited. 
which shows that $0=\operatorname{div}_{y}(\boldsymbol{R} v(y))=\operatorname{div}_{y}\left(v(y) \boldsymbol{R}^{*}\right)=\frac{\partial v_{l}}{\partial y_{\tau}} \boldsymbol{R}_{\tau l}$ in the sense of distributions because $\phi \in C_{\#}^{\infty}\left(Y^{\mathrm{m}}\right)$ is arbitrary.

Using standard mollification techniques with a $Y^{\mathrm{m}}$-periodic, smooth kernel, we may construct a sequence $\left\{v_{h}\right\}_{h \in \mathbb{N}} \subset C_{\#}^{\infty}\left(Y^{\mathrm{m}} ; \mathbb{R}^{\mathrm{m}}\right)$ such that $\operatorname{div}_{y}\left(v_{h}(y) \boldsymbol{R}^{*}\right)=0$ in $Y^{\mathrm{m}}$ and $\lim _{h \rightarrow \infty}\left\|v_{h}-v\right\|_{L^{p^{\prime}}\left(Y^{\mathrm{m}} ; \mathbb{R}^{\mathrm{n}}\right)}=0$. Then, by (5.3) with $\psi=v_{h}$ and by the Lebesgue dominated convergence theorem, we obtain $\int_{Y^{\mathrm{m}}} w_{0}(y) \cdot v(y) \mathrm{d} y=0$, which contradicts (5.5). Thus, $w_{0} \in \mathcal{G}_{\boldsymbol{R}}^{p}$.

Proposition 5.5. Let $\left\{u_{\varepsilon}\right\}_{\varepsilon} \subset W^{1, p}(\Omega)$ be a bounded sequence, and assume that $\boldsymbol{R}$ satisfies (1.2). Then, there exist a subsequence $\varepsilon^{\prime} \preceq \varepsilon$ and functions $u \in W^{1, p}(\Omega)$ and $w \in L^{p}\left(\Omega ; \mathcal{G}_{\boldsymbol{R}}^{p}\right)$ such that

$$
u_{\varepsilon^{\prime}} \stackrel{\boldsymbol{R}-2 s c}{\longrightarrow} u \quad \text { and } \nabla u_{\varepsilon^{\prime}} \stackrel{\boldsymbol{R}-2 s c}{\longrightarrow} \nabla u+w .
$$

Proof. By the reflexibility of $W^{1, p}(\Omega)$ and Proposition 3.9, there exist $u \in W^{1, p}(\Omega)$, $u_{0} \in L^{p}\left(\Omega \times Y^{\mathrm{m}}\right)$, and $U_{0} \in L^{p}\left(\Omega \times Y^{\mathrm{m}} ; \mathbb{R}^{\mathrm{m}}\right)$ such that, extracting a subsequence if necessary,

$$
u_{\varepsilon} \rightarrow u \text { weakly in } W^{1, p}(\Omega), u_{\varepsilon} \stackrel{\boldsymbol{R}-2 s c}{\longrightarrow} u_{0}, \text { and } \nabla u_{\varepsilon} \stackrel{\boldsymbol{R}-2 s c}{\longrightarrow} U_{0} .
$$

By the Rellich-Kondrachov theorem, $u_{\varepsilon} \rightarrow u$ in $L^{p}(\Omega)$. Hence, Proposition 3.5 and the uniqueness of the $\boldsymbol{R}$-two-scale limit (see Remark 3.2) yield

$$
u \equiv u_{0}
$$

We are left to prove that $U_{0}$ in (5.6) is of the form $U_{0}(x, y)=\nabla u(x)+w(x, y)$ for some $w \in L^{p}\left(\Omega ; \mathcal{G}_{\boldsymbol{R}}\right)$. Let $\Phi \in C_{c}^{\infty}\left(\Omega ; C_{\#}^{\infty}\left(Y^{\mathrm{m}} ; \mathbb{R}^{\mathrm{n}}\right)\right)$ be such that $\frac{\partial \Phi_{l}}{\partial y_{\tau}} \boldsymbol{R}_{\tau l}=0$ in $\Omega \times Y^{\mathrm{m}}, l \in\{1, \ldots, \mathrm{m}\}, \tau \in\{1, \ldots, \mathrm{m}\}$. Then, using integration by parts, the second convergence in (5.6), and the fact that $u_{0} \equiv u \in W^{1, p}(\Omega)$, we obtain

$$
\begin{aligned}
& \int_{\Omega \times Y^{\mathrm{m}}} U_{0}(x, y) \cdot \Phi(x, y) \mathrm{d} x \mathrm{~d} y \\
& =\lim _{\varepsilon \rightarrow 0^{+}} \int_{\Omega} \nabla u_{\varepsilon}(x) \cdot \Phi\left(x, \frac{\boldsymbol{R} x}{\varepsilon}\right) \mathrm{d} x \\
& =-\lim _{\varepsilon \rightarrow 0^{+}}\left(\int_{\Omega} u_{\varepsilon}(x) \operatorname{div}_{x} \Phi\left(x, \frac{\boldsymbol{R} x}{\varepsilon}\right) \mathrm{d} x+\frac{1}{\varepsilon} \int_{\Omega} u_{\varepsilon}(x) \frac{\partial \Phi_{l}}{\partial y_{\tau}}\left(x, \frac{\boldsymbol{R} x}{\varepsilon}\right) \boldsymbol{R}_{\tau l} \mathrm{~d} x\right) \\
& =-\lim _{\varepsilon \rightarrow 0^{+}} \int_{\Omega} u_{\varepsilon}(x) \operatorname{div}_{x} \Phi\left(x, \frac{\boldsymbol{R} x}{\varepsilon}\right) \mathrm{d} x=-\int_{\Omega \times Y^{\mathrm{m}}} u(x) \operatorname{div}_{x} \Phi(x, y) \mathrm{d} x \mathrm{~d} y \\
& =\int_{\Omega \times Y^{\mathrm{m}}} \nabla u(x) \cdot \Phi(x, y) \mathrm{d} x \mathrm{~d} y .
\end{aligned}
$$

Hence,

$$
\int_{\Omega \times Y^{\mathrm{m}}}\left(U_{0}(x, y)-\nabla u(x)\right) \cdot \Phi(x, y) \mathrm{d} x \mathrm{~d} y=0
$$

for all $\Phi \in C_{c}^{\infty}\left(\Omega ; C_{\#}^{\infty}\left(Y^{\mathrm{m}} ; \mathbb{R}^{\mathrm{n}}\right)\right)$ such that $\frac{\partial \Phi_{l}}{\partial y_{\tau}} \boldsymbol{R}_{\tau l}=0$ in $\Omega \times Y^{\mathrm{m}}$. Invoking Lemma 5.4, we conclude for a.e.- $x \in \Omega$ that $U_{0}(x, \cdot)-\nabla u(x) \in \mathcal{G}_{\boldsymbol{R}}^{p}$, which, together with the fact that the map $(x, y) \mapsto U_{0}(x, y)-\nabla u(x)$ belongs to $L^{p}\left(\Omega \times Y^{\mathrm{m}} ; \mathbb{R}^{\mathrm{n}}\right)$, concludes the proof. 
The next proposition shows that Proposition 5.5 fully characterizes the $\boldsymbol{R}$-twoscale limit of bounded sequences in $W^{1, p}(\Omega)$. To the best of our knowledge, in the framework of $\boldsymbol{R}$-two-scale convergence, this result is new in the literature even for $p=2$.

Proposition 5.6. Let $u \in W^{1, p}(\Omega)$ and $w \in L^{p}\left(\Omega ; \mathcal{G}_{\boldsymbol{R}}^{p}\right)$, and assume that $\boldsymbol{R}$ satisfies (1.2). Then, there exists a bounded sequence $\left\{u_{\varepsilon}\right\}_{\varepsilon} \subset W^{1, p}(\Omega)$ such that

$$
u_{\varepsilon} \stackrel{\boldsymbol{R}-2 s c}{\longrightarrow} u \text { and } \nabla u_{\varepsilon} \stackrel{\boldsymbol{R}-2 s c}{\longrightarrow} \nabla u+w .
$$

Proof. We first consider the case in which $w \in W^{1, p}\left(\Omega ; \mathcal{G}_{\boldsymbol{R}}^{p}\right)$.

For each $k \in \mathbb{Z}^{\mathrm{m}}$, define

$$
\hat{w}_{k}(x):=\int_{Y^{\mathrm{m}}} w(x, y) e^{-2 \pi i k \cdot y} \mathrm{~d} y, \quad x \in \Omega .
$$

Because $w \in W^{1, p}\left(\Omega ; \mathcal{G}_{R}^{p}\right)$, we have $\hat{w}_{k} \in W^{1, p}\left(\Omega ; \mathbb{C}^{n}\right)$ with $\hat{w}_{0} \equiv 0$; moreover, for a.e.- $x \in \Omega$, we have $\hat{w}_{k}(x)=\lambda_{k}(x) \boldsymbol{R}^{*} k$ for some $\lambda_{k} \in W^{1, p}(\Omega ; \mathbb{C})$ with $\lambda_{0} \equiv 0$.

For each $N \in \mathbb{N}$, let $\tilde{w}_{N} \in W^{1, p}\left(\Omega ; C_{\#}^{\infty}\left(Y^{\mathrm{m}} ; \mathbb{C}\right)\right)$ be the function defined by

$$
\tilde{w}_{N}(x, y):=\sum_{\substack{k \in \mathbb{Z}^{\mathrm{m}} \\|k|_{\infty} \leqslant N}} \frac{1}{2 \pi i} \lambda_{k}(x) e^{2 \pi i k \cdot y}, \quad(x, y) \in \Omega \times \mathbb{R}^{\mathrm{m}} .
$$

Note that the function

$$
\begin{aligned}
w_{N}(x, y) & :=\boldsymbol{R}^{*} \nabla_{y} \tilde{w}_{N}(x, y)=\sum_{\substack{k \in \mathbb{Z}^{\mathrm{m}} \\
|k| \infty \leqslant N}} \lambda_{k}(x) \boldsymbol{R}^{*} k e^{2 \pi i k \cdot y} \\
& =\sum_{\substack{k \in \mathbb{Z}^{\mathrm{m}} \\
|k| \infty \leqslant N}} \hat{w}_{k}(x) e^{2 \pi i k \cdot y}, \quad(x, y) \in \Omega \times \mathbb{R}^{\mathrm{m}},
\end{aligned}
$$

belongs to $W^{1, p}\left(\Omega ; C_{\#}^{\infty}\left(Y^{\mathrm{m}} ; \mathbb{R}^{\mathrm{m}}\right)\right)$ and, by Remark 5.2 , satisfies

$$
\lim _{N \rightarrow \infty} \int_{\Omega \times Y_{\mathrm{m}}}\left|w_{N}(x, y)-w(x, y)\right|^{p} \mathrm{~d} x \mathrm{~d} y .
$$

Finally, for each $\varepsilon$, we define $\bar{w}_{N}:=\operatorname{Re}\left(\tilde{w}_{N}\right)$ and

$$
u_{\varepsilon, N}(x):=u(x)+\varepsilon \bar{w}_{N}\left(x, \frac{\boldsymbol{R} x}{\varepsilon}\right), x \in \Omega .
$$

Then, $u_{\varepsilon, N} \in W^{1, p}(\Omega)$ with

$$
\left\|u_{\varepsilon, N}\right\|_{L^{p}(\Omega)} \leqslant\|u\|_{L^{p}(\Omega)}+\varepsilon\left\|\bar{w}_{N}\right\|_{L^{p}\left(\Omega ; C_{\#}\left(Y^{\mathrm{m}}\right)\right)}
$$

and

$\left\|\nabla u_{\varepsilon, N}\right\|_{L^{p}\left(\Omega ; \mathbb{R}^{\mathrm{n}}\right)} \leqslant\|\nabla u\|_{L^{p}\left(\Omega ; \mathbb{R}^{\mathrm{n}}\right)}+\varepsilon\left\|\nabla_{x} \bar{w}_{N}\right\|_{L^{p}\left(\Omega ; C_{\#}\left(Y^{\mathrm{m}} ; \mathbb{R}^{\mathrm{n}}\right)\right)}+\left\|w_{N}\right\|_{L^{p}\left(\Omega ; C_{\#}\left(Y^{\mathrm{m}} ; \mathbb{R}^{\mathrm{n}}\right)\right)}$. 
Let $\varphi \in L^{p^{\prime}}\left(\Omega ; C_{\#}\left(Y^{\mathrm{m}}\right)\right)$ and $\Phi \in L^{p^{\prime}}\left(\Omega ; C_{\#}\left(Y^{\mathrm{m}} ; \mathbb{R}^{\mathrm{m}}\right)\right)$. By Proposition 3.7 , we have

$$
\begin{aligned}
& \lim _{N \rightarrow \infty} \lim _{\varepsilon \rightarrow 0^{+}} \int_{\Omega} u_{\varepsilon, N}(x) \varphi\left(x, \frac{\boldsymbol{R} x}{\varepsilon}\right) \mathrm{d} x \\
& =\lim _{N \rightarrow \infty} \lim _{\varepsilon \rightarrow 0^{+}} \int_{\Omega}\left(u(x)+\varepsilon \bar{w}_{N}\left(x, \frac{\boldsymbol{R} x}{\varepsilon}\right)\right) \varphi\left(x, \frac{\boldsymbol{R} x}{\varepsilon}\right) \mathrm{d} x \\
& =\int_{\Omega \times Y^{\mathrm{m}}} u(x) \varphi(x, y) \mathrm{d} x \mathrm{~d} y
\end{aligned}
$$

and, also using (5.7),

$$
\begin{aligned}
& \lim _{N \rightarrow \infty} \lim _{\varepsilon \rightarrow 0^{+}} \int_{\Omega} \nabla u_{\varepsilon, N}(x) \cdot \Phi\left(x, \frac{\boldsymbol{R} x}{\varepsilon}\right) \mathrm{d} x \\
& \quad=\lim _{N \rightarrow \infty} \lim _{\varepsilon \rightarrow 0^{+}} \int_{\Omega}\left(\nabla u(x)+\varepsilon \nabla_{x} \bar{w}\left(x, \frac{\boldsymbol{R} x}{\varepsilon}\right)+w_{N}\left(x, \frac{\boldsymbol{R} x}{\varepsilon}\right)\right) \cdot \Phi\left(x, \frac{\boldsymbol{R} x}{\varepsilon}\right) \mathrm{d} x \\
& \quad=\int_{\Omega \times Y^{\mathrm{m}}}(\nabla u(x)+w(x, y)) \cdot \Phi(x, y) \mathrm{d} x \mathrm{~d} y .
\end{aligned}
$$

Due to the separability of $L^{p^{\prime}}\left(\Omega ; C_{\#}\left(Y^{\mathrm{m}}\right)\right)$ and $L^{p^{\prime}}\left(\Omega ; C_{\#}\left(Y^{\mathrm{m}} ; \mathbb{R}^{\mathrm{m}}\right)\right)$ and $(5.8)-$ (5.9), we can proceed as in [29, proof of Prop. 1.11, p. 449] to find a sequence $\left\{N_{\varepsilon}\right\}_{\varepsilon}$ such that $N_{\varepsilon} \rightarrow \infty$ as $\varepsilon \rightarrow 0^{+}$and $\tilde{u}_{\varepsilon}:=u_{\varepsilon, N_{\varepsilon}} \in W^{1, p}(\Omega)$ satisfies

$$
\lim _{\varepsilon \rightarrow 0^{+}} \int_{\Omega} \tilde{u}_{\varepsilon}(x) \varphi\left(x, \frac{\boldsymbol{R} x}{\varepsilon}\right) \mathrm{d} x=\int_{\Omega \times Y^{\mathrm{m}}} u(x) \varphi(x, y) \mathrm{d} x \mathrm{~d} y
$$

and

$$
\lim _{\varepsilon \rightarrow 0^{+}} \int_{\Omega} \nabla \tilde{u}_{\varepsilon}(x) \cdot \Phi\left(x, \frac{\boldsymbol{R} x}{\varepsilon}\right) \mathrm{d} x=\int_{\Omega \times Y^{\mathrm{m}}}(\nabla u(x)+w(x, y)) \cdot \Phi(x, y) \mathrm{d} x \mathrm{~d} y
$$

for all $\varphi \in L^{p^{\prime}}\left(\Omega ; C_{\#}\left(Y^{\mathrm{m}}\right)\right)$ and $\Phi \in L^{p^{\prime}}\left(\Omega ; C_{\#}\left(Y^{\mathrm{m}} ; \mathbb{R}^{\mathrm{m}}\right)\right)$; that is,

$$
\tilde{u}_{\varepsilon} \stackrel{\boldsymbol{R}-2 s c}{\longrightarrow} u \text { and } \nabla \tilde{u}_{\varepsilon} \stackrel{\boldsymbol{R}-2 s c}{\longrightarrow} \nabla u+w .
$$

The boundedness of $\tilde{u}_{\varepsilon}$ in $W^{1, p}(\Omega)$ follows from Proposition 3.4.

To conclude, we treat the general case in which $w \in L^{p}\left(\Omega ; \mathcal{G}_{\boldsymbol{R}}^{p}\right)$. We claim that there exists a sequence $\left\{\bar{w}_{N}\right\}_{N \in \mathbb{N}} \subset W^{1, p}\left(\Omega ; \mathcal{G}_{\boldsymbol{R}}^{p}\right)$ such that

$$
\lim _{N \rightarrow \infty}\left\|\bar{w}_{N}-w\right\|_{L^{p}\left(\Omega \times Y^{\mathrm{m}} ; \mathbb{R}^{\mathrm{n}}\right)}=0 .
$$

Assume that the claim holds. Then, by the previous case, for each $N \in \mathbb{N}$, there exists a bounded sequence $\left\{u_{\varepsilon}^{N}\right\}_{\varepsilon} \subset W^{1, p}(\Omega)$ such that

$$
u_{\varepsilon}^{N} \stackrel{R-2 s c}{\longrightarrow} u \text { and } \nabla u_{\varepsilon}^{N} \stackrel{R-2 s c}{\longrightarrow} \nabla u+\bar{w}_{N}
$$

as $\varepsilon \rightarrow 0$.

Let $\varphi \in L^{p^{\prime}}\left(\Omega ; C_{\#}\left(Y^{\mathrm{m}}\right)\right)$ and $\Phi \in L^{p^{\prime}}\left(\Omega ; C_{\#}\left(Y^{\mathrm{m}} ; \mathbb{R}^{\mathrm{m}}\right)\right)$. Using (5.11) first, and then (5.10), we obtain

$$
\lim _{N \rightarrow \infty} \lim _{\varepsilon \rightarrow 0^{+}} \int_{\Omega} u_{\varepsilon}^{N}(x) \varphi\left(x, \frac{\boldsymbol{R} x}{\varepsilon}\right) \mathrm{d} x=\int_{\Omega \times Y^{\mathrm{m}}} u(x) \varphi(x, y) \mathrm{d} x \mathrm{~d} y
$$

Copyright $@$ by SIAM. Unauthorized reproduction of this article is prohibited. 
and

$$
\begin{aligned}
\lim _{N \rightarrow \infty} \lim _{\varepsilon \rightarrow 0^{+}} \int_{\Omega} \nabla u_{\varepsilon}^{N}(x) \cdot \Phi\left(x, \frac{\boldsymbol{R} x}{\varepsilon}\right) \mathrm{d} x & =\lim _{N \rightarrow \infty} \int_{\Omega \times Y_{\mathrm{m}}^{\mathrm{m}}}\left(\nabla u(x)+\bar{w}_{N}(x, y)\right) \cdot \Phi(x, y) \mathrm{d} x \mathrm{~d} y \\
& =\int_{\Omega \times Y^{\mathrm{m}}}(\nabla u(x)+w(x, y)) \cdot \Phi(x, y) \mathrm{d} x \mathrm{~d} y .
\end{aligned}
$$

Finally, arguing as in the previous case, we can find a sequence $\left\{N_{\varepsilon}\right\}_{\varepsilon}$ such that $N_{\varepsilon} \rightarrow \infty$ as $\varepsilon \rightarrow 0^{+}$, and $\tilde{u}_{\varepsilon}:=u_{\varepsilon}^{N_{\varepsilon}} \in W^{1, p}(\Omega)$ satisfies the requirements.

We are left to prove (5.10). As before, for each $k \in \mathbb{Z}^{\mathrm{m}}$, define

$$
\hat{w}_{k}(x):=\int_{Y^{\mathrm{m}}} w(x, y) e^{-2 \pi i k \cdot y} \mathrm{~d} y, \quad x \in \Omega .
$$

Because $w \in L^{p}\left(\Omega ; \mathcal{G}_{\boldsymbol{R}}^{p}\right)$, we have $\hat{w}_{k} \in L^{p}\left(\Omega ; \mathbb{C}^{\mathrm{n}}\right)$ with $\hat{w}_{0} \equiv 0$; moreover, for a.e.$x \in \Omega$, we have $\hat{w}_{k}(x)=\lambda_{k}(x) \boldsymbol{R}^{*} k$ for some $\lambda_{k} \in L^{p}(\Omega ; \mathbb{C})$ with $\lambda_{0} \equiv 0$. Then, for each $k \in \mathbb{Z}^{\mathrm{m}}$, we can find a sequence $\left\{\lambda_{k}^{j}\right\}_{j \in \mathbb{N}} \subset W^{1, p}(\Omega ; \mathbb{C})$, with $\lambda_{0}^{j}=0$, such that $\lambda_{k}^{j} \rightarrow \lambda_{k}$ in $L^{p}(\Omega ; \mathbb{C})$ as $j \rightarrow \infty$. In particular, we have

$$
\lim _{j \rightarrow \infty}\left(\int_{\Omega}\left|\lambda_{k}^{j}(x) \boldsymbol{R}^{*} k-\lambda_{k}(x) \boldsymbol{R}^{*} k\right|^{p} \mathrm{~d} x\right)^{\frac{1}{p}}=\lim _{j \rightarrow \infty}\left|\boldsymbol{R}^{*} k\right|\left(\int_{\Omega}\left|\lambda_{k}^{j}(x)-\lambda_{k}(x)\right|^{p} \mathrm{~d} x\right)^{\frac{1}{p}}=0 .
$$

Fix $N \in \mathbb{N}$; by (5.12), there exists $j_{N} \in \mathbb{N}$ such that

$$
\sum_{\substack{k \in \mathbb{Z}^{\mathrm{m}} \\|k| \infty \leqslant \leqslant}}\left(\int_{\Omega}\left|\lambda_{k}^{j_{N}}(x) \boldsymbol{R}^{*} k-\lambda_{k}(x) \boldsymbol{R}^{*} k\right|^{p} \mathrm{~d} x\right)^{\frac{1}{p}} \leqslant \frac{1}{N} .
$$

Defining

$$
\tilde{w}_{N}(x, y):=\sum_{\substack{k \in \mathbb{Z}^{\mathrm{m}} \\|k| \infty \leqslant N}} \lambda_{k}^{j_{N}}(x) \boldsymbol{R}^{*} k e^{2 \pi i k \cdot y} \quad \text { and } \quad \bar{w}_{N}:=\operatorname{Re}\left(\tilde{w}_{N}\right),
$$

we have $\bar{w}_{N} \in W^{1, p}\left(\Omega ; \mathcal{G}_{\boldsymbol{R}}^{p}\right)$; moreover, invoking Remark 5.2 and (5.13), we have

$$
\begin{aligned}
& \limsup _{N \rightarrow \infty}\left(\int_{\Omega \times Y^{\mathrm{m}}}\left|\bar{w}_{N}(x, y)-w(x, y)\right|^{p} \mathrm{~d} x \mathrm{~d} y\right)^{\frac{1}{p}} \\
& \leqslant \limsup _{N \rightarrow \infty}\left(\int_{\Omega \times Y^{\mathrm{m}}}\left|\tilde{w}_{N}(x, y)-w(x, y)\right|^{p} \mathrm{~d} x \mathrm{~d} y\right)^{\frac{1}{p}} \\
& \leqslant \limsup _{N \rightarrow \infty}\left[\left(\int_{\Omega \times Y_{\mathrm{m}}}\left|\tilde{w}_{N}(x, y)-w_{N}(x, y)\right|^{p} \mathrm{~d} x \mathrm{~d} y\right)^{\frac{1}{p}}\right. \\
& \left.\quad+\left(\int_{\Omega \times Y^{\mathrm{m}}}\left|w_{N}(x, y)-w(x, y)\right|^{p} \mathrm{~d} x \mathrm{~d} y\right)^{\frac{1}{p}}\right] \\
& =\limsup _{N \rightarrow \infty}\left(\int_{\Omega \times Y^{\mathrm{m}}}\left|\sum_{\substack{k \in \mathbb{Z}^{\mathrm{m}} \\
|k| \infty \leqslant N}}\left(\lambda_{k}^{j_{N}}(x)-\lambda_{k}(x)\right) \boldsymbol{R}^{*} k e^{2 \pi i k \cdot y}\right|^{p} \mathrm{~d} x \mathrm{~d} y\right)^{\frac{1}{p}} \leqslant \limsup _{N \rightarrow \infty} \frac{1}{N}=0,
\end{aligned}
$$

which concludes the proof of (5.10). 
Remark 5.7. Let $\Omega \subset \mathbb{R}^{\mathrm{n}}$ be a simply connected, bounded, and open set. Applying Proposition 5.6 to $u=0$ and $w \in \mathcal{G}_{\boldsymbol{R}}^{p}$, we can find a bounded sequence $\left\{u_{\varepsilon}\right\}_{\varepsilon} \subset W^{1, p}(\Omega)$ such that

$$
u_{\varepsilon} \stackrel{\boldsymbol{R}-2 s c}{\longrightarrow} 0 \text { and } \nabla u_{\varepsilon} \stackrel{\boldsymbol{R}-2 s c}{\longrightarrow} w
$$

Then, by Proposition 3.4, we have $u_{\varepsilon} \rightarrow 0$ in $W^{1, p}(\Omega)$ and $\int_{Y^{\mathrm{m}}} w(y) \mathrm{d} y=0$. On the other hand, using the uniqueness of the $\boldsymbol{R}$-two-scale limit (see Remark 3.2) and Proposition 3.14 with $\mathbb{d}=\mathrm{m}$ and $\mathcal{A}=$ curl in $\mathbb{R}^{\mathrm{m}}$, we conclude that $w \in L_{\#}^{p}\left(Y^{\mathrm{m}} ; \mathbb{R}^{\mathrm{m}}\right)$

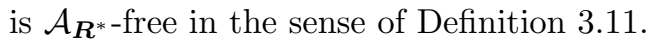

Conversely, if $w \in L_{\#}^{p}\left(Y^{\mathrm{m}} ; \mathbb{R}^{\mathrm{n}}\right)$ is $\mathcal{A}_{\boldsymbol{R}^{*}}$-free with $\int_{Y^{\mathrm{m}}} w(y) \mathrm{d} y=0$, then by Proposition 3.15 there exists a bounded and $\mathcal{A}$-free sequence, $\left\{u_{\varepsilon}\right\}_{\varepsilon}$, in $L^{p}\left(\Omega ; \mathbb{R}^{\mathrm{n}}\right)$ such that $u_{\varepsilon} \stackrel{R-2 s c}{\longrightarrow} u$. As we are in the $\mathcal{A}=$ curl case and $\Omega$ is simply connected, we can find a bounded sequence, $\left\{v_{\varepsilon}\right\}_{\varepsilon}$, in $W^{1, p}(\Omega)$ such that $\int_{\Omega} v_{\varepsilon}(x) \mathrm{d} x=0$ and $\nabla v_{\varepsilon}=u_{\varepsilon}$. Then, by Proposition 3.4, we deduce that $v_{\varepsilon} \rightarrow 0$ in $W^{1, p}(\Omega)$. Finally, Remark 3.2 and Proposition 5.5 yield $w \in \mathcal{G}_{\boldsymbol{R}}^{p}$.

Thus, in the $\mathcal{A}=$ curl case in $\mathbb{R}^{\mathrm{n}}$, we have that $w \in L_{\#}^{p}\left(Y^{\mathrm{m}} ; \mathbb{R}^{\mathrm{n}}\right)$ is $\mathcal{A}_{\boldsymbol{R}^{*}}$-free in the sense of Definition 3.11 if and only if $w \in \mathcal{G}_{\boldsymbol{R}}^{p}$.

To conclude, we observe that Theorem 1.4 is an immediate consequence of the previous results.

Proof of Theorem 1.4. The claim in Theorem 1.4 follows from Propositions 5.5 and 5.6.

\section{REFERENCES}

[1] G. Allaire, Homogenization and two-scale convergence, SIAM J. Math. Anal., 23 (1992), pp. 1482-1518, https://doi.org/10.1137/0523084.

[2] G. Allaire and M. Briane, Multiscale convergence and reiterated homogenisation, Proc. Roy. Soc. Edinburgh Sect. A, 126 (1996), pp. 297-342.

[3] M. AmaR, Two-scale convergence and homogenization on $B V(\Omega)$, Asymptot. Anal., 16 (1998), pp. $65-84$.

[4] A. A. Azuha, A. Klimkowicz, And A. TAkasaki, Quaternary quasicrystal alloys for hydrogen storage technology, MRS Advances, 5 (2020), pp. 1071-1083, https://doi.org/10.1557/adv. 2020.96.

[5] M. BAÍA AND I. FONSECA, The limit behavior of a family of variational multiscale problems, Indiana Univ. Math. J., 56 (2007), pp. 1-50, https://doi.org/10.1512/iumj.2007.56.2869.

[6] P. D. Bloom, K. Baikerikar, J. U. Otaigbe, and V. V. Sheares, Development of novel polymer/quasicrystal composite materials, Mater. Sci. Engrg. A, 294-296 (2000), pp. 156159, https://doi.org/10.1016/S0921-5093(00)01230-2.

[7] E. BombieRI AND J. E. TAYLOR, Quasicrystals, tilings, and algebraic number theory: Some preliminary connections, in The Legacy of Sonya Kovalevskaya (Cambridge, Mass., and Amherst, Mass., 1985), Contemp. Math. 64, Amer. Math. Soc., Providence, RI, 1987, pp. 241-264, https://doi.org/10.1090/conm/064/881466.

[8] G. Bouchitté, S. Guenneau, and F. Zolla, Homogenization of dielectric photonic quasi crystals, Multiscale Model. Simul., 8 (2010), pp. 1862-1881, https://doi.org/10.1137/ 090770333.

[9] A. Braides, Homogenization of some almost periodic coercive functional, Rend. Accad. Naz. Sci. XL Mem. Mat. (5), 9 (1985), pp. 313-321.

[10] A. Braides, A homogenization theorem for weakly almost periodic functionals, Rend. Accad. Naz. Sci. XL Mem. Mat. (5), 10 (1986), pp. 261-281.

[11] A. Braides, Homogenization of weakly almost-periodic functionals, Atti Accad. Naz. Lincei Rend. Cl. Sci. Fis. Mat. Nat. (8), 81 (1987), pp. 29-33.

[12] A. Braides, Almost periodic methods in the theory of homogenization, Appl. Anal., 47 (1992), pp. 259-277, https://doi.org/10.1080/00036819208840144. 
[13] A. Braides, I. Fonseca, And G. Leoni, A-quasiconvexity: Relaxation and homogenization, ESAIM Control Optim. Calc. Var., 5 (2000), pp. 539-577, https://doi.org/10.1051/cocv: 2000121.

[14] H. Brezis, Analyse fonctionnelle: Théorie et applications, Collection Mathématiques Appliquées pour la Maîtrise [Collection of Applied Mathematics for the Master's Degree], Masson, Paris, 1983.

[15] L. Bufford and I. Fonseca, A note on two scale compactness for $p=1$, Port. Math., 72 (2015), pp. 101-117, https://doi.org/10.4171/PM/1959.

[16] J. W. CAhn and J. E. TAYlor, An introduction to quasicrystals, in The Legacy of Sonya Kovalevskaya (Cambridge, Mass., and Amherst, Mass., 1985), Contemp. Math. 64, Amer. Math. Soc., Providence, RI, 1987, pp. 265-286, https://doi.org/10.1090/conm/064/881467.

[17] J. CaSAdo-Díaz And I. Gayte, The two-scale convergence method applied to generalized Besicovitch spaces, Proc. R. Soc. Lond. Ser. A, 458 (2002), pp. 2925-2946, https://doi.org/10. 1098/rspa.2002.1003.

[18] C. Castaing and M. Valadier, Convex Analysis and Measurable Multifunctions, Lecture Notes in Math. 580, Springer-Verlag, Berlin, 1977.

[19] D. Cioranescu, A. Damlamian, and R. De Arcangelis, Homogenization of nonlinear integrals via the periodic unfolding method, C. R. Math. Acad. Sci. Paris Ser. I, 339 (2004), pp. 77-82, https://doi.org/10.1016/j.crma.2004.03.028.

[20] B. Dacorogna, Quasiconvexity and relaxation of nonconvex problems in the calculus of variations, J. Funct. Anal., 46 (1982), pp. 102-118, https://doi.org/10.1016/0022-1236(82) 90046-5.

[21] B. Dacorogna, Weak Continuity and Weak Lower Semicontinuity of Nonlinear Functionals, Lecture Notes in Math. 922, Springer-Verlag, Berlin, 1982.

[22] G. Dal Maso, An Introduction to $\Gamma$-Convergence, Progr. Nonlinear Differential Equations Appl. 8, Birkhäuser Boston Inc., Boston, MA, 1993.

[23] E. Davoli And I. Fonseca, Homogenization of integral energies under periodically oscillating differential constraints, Calc. Var. Partial Differential Equations, 55 (2016), 69, https: //doi.org/10.1007/s00526-016-0988-5.

[24] E. Davoli and I. Fonseca, Periodic homogenization of integral energies under space-dependent differential constraints, Port. Math., 73 (2016), pp. 279-317, https://doi.org/10.4171/PM/ 1988.

[25] R. De Arcangelis, Homogenization of Besicovitch type almost periodic functionals, Appl. Anal., 43 (1992), pp. 77-98, https://doi.org/10.1080/00036819208840053.

[26] N. G. DE BruiJn, Algebraic theory of Penrose's non-periodic tilings of the plane I, II, Indag. Math., 84 (1981), pp. 39-52; 53-66, https://doi.org/10.1016/1385-7258(81)90016-0; https: //doi.org/10.1016/1385-7258(81)90017-2.

[27] M. Duneau and A. Katz, Quasiperiodic patterns, Phys. Rev. Lett., 54 (1985), pp. 2688-2691, https://doi.org/10.1103/PhysRevLett.54.2688.

[28] M. Engel, P. F. Damasceno, C. L. Phillips, and S. C. Glotzer, Computational selfassembly of a one-component icosahedral quasicrystal, Nature Materials, 14 (2015), pp. 109-116, https://doi.org/10.1038/nmat4152.

[29] R. FERREIRA AND I. FONSECA, Characterization of the multiscale limit associated with bounded sequences in BV, J. Convex Anal., 19 (2012), pp. 403-452.

[30] R. Ferreira and I. Fonseca, Reiterated homogenization in $B V$ via multiscale convergence, SIAM J. Math. Anal., 44 (2012), pp. 2053-2098, https://doi.org/10.1137/110826205.

[31] I. FonseCA AND S. KRÖMER, Multiple integrals under differential constraints: Two-scale convergence and homogenization, Indiana Univ. Math. J., 59 (2010), pp. 427-458, https: //doi.org/10.1512/iumj.2010.59.4249.

[32] I. FonsecA AND S. MÜLlER, A-quasiconvexity, lower semicontinuity, and Young measures, SIAM J. Math. Anal., 30 (1999), pp. 1355-1390, https://doi.org/10.1137/ S0036141098339885.

[33] I. Fonseca And E. Zappale, Multiscale relaxation of convex functionals, J. Convex Anal., 10 (2003), pp. 325-350.

[34] L. Grafakos, Classical Fourier Analysis, 3rd ed., Grad. Texts Math. 249, Springer, New York, 2014, https://doi.org/10.1007/978-1-4939-1194-3.

[35] D. Gratias, A. Katz, and M. Quiquandon, Geometry of approximant structures in quasicrystals, J. Phys., 7 (1995), pp. 9101-9125.

[36] J. Guo, T. Sun, AND E. PAN, Three-dimensional nonlocal buckling of composite nanoplates with coated one-dimensional quasicrystal in an elastic medium, Int. J. Solids Structures, 185-186 (2020), pp. 272-280, https://doi.org/10.1016/j.ijsolstr.2019.08.033.

Copyright $@$ by SIAM. Unauthorized reproduction of this article is prohibited. 
[37] M. Heida, S. Neukamm, and M. Varga, Stochastic Unfolding and Homogenization, preprint, https://arxiv.org/abs/1805.09546, 2018.

[38] K. Kamiya, T. Takeuchi, N. Kabeya, N. Wada, T. Ishimasa, A. Ochiai, K. Deguchi, K. ImUra, And N. K. Sato, Discovery of superconductivity in quasicrystal, Nature Commun., 9 (2018), 154, https://doi.org/10.1038/s41467-017-02667-x.

[39] S. Kenzari, D. Bonina, J. Dubois, And V. Fournée, Quasicrystal-polymer composites for selective laser sintering technology, Mater. Design, 35 (2012), pp. 691-695, https://doi. org/10.1016/j.matdes.2011.10.032.

[40] S. M. KozLOv, The averaging of random operators, Mat. Sb. (N.S.), 109(151) (1979), pp. 188202, 327 (in Russian), https://doi.org/10.1070/SM1980v037n02ABEH001948.

[41] C. Kreisbeck and S. Krömer, Heterogeneous thin films: Combining homogenization and dimension reduction with directors, SIAM J. Math. Anal., 48 (2016), pp. 785-820, https: //doi.org/10.1137/15M1032557.

[42] J. C. LAGARIAS, Mathematical quasicrystals and the problem of diffraction, in Directions in Mathematical Quasicrystals, CRM Monogr. Ser. 13, Amer. Math. Soc., Providence, RI, 2000, pp. 61-93.

[43] D. Levine and P. J. Steinhardt, Quasicrystals: A new class of ordered structures, Phys. Rev. Lett., 53 (1984), pp. 2477-2480, https://doi.org/10.1103/PhysRevLett.53.2477.

[44] D. Lukkassen, G. Nguetseng, and P. Wall, Two-scale convergence, Int. J. Pure Appl. Math., 2 (2002), pp. 33-81, https://ijpam.eu/contents/2002-2-1/3/3.pdf.

[45] J. Matias, M. Morandotti, And P. M. Santos, Homogenization of functionals with linear growth in the context of $\mathcal{A}$-quasiconvexity, Appl. Math. Optim., 72 (2015), pp. 523-547, https://doi.org/10.1007/s00245-015-9289-1.

[46] Y. Meyer, Quasicrystals, Diophantine approximation and algebraic numbers, in Beyond Quasicrystals, Springer, Berlin, 1995, pp. 3-16.

[47] L. Modica and G. Dal Maso, Nonlinear stochastic homogenization and ergodic theory, J. Reine Angew. Math., no. 368, 1986, pp. 28-42, https://doi.org/10.1515/crll.1986.368.28.

[48] F. Murat, Compacité par compensation: Condition nécessaire et suffisante de continuité faible sous une hypothèse de rang constant, Ann. Scuola Norm. Sup. Pisa Cl. Sci. (4), 8 (1981), pp. 69-102, http://www.numdam.org/item/ASNSP_1981_4_8_1_69_0/.

[49] Y. Nagaoka, H. Zhu, D. EgGert, and O. Chen, Single-component quasicrystalline nanocrystal superlattices through flexible polygon tiling rule, Science, 362 (2018), pp. 1396-1400, https://doi.org/10.1126/science.aav0790.

[50] S. Neukamm, Rigorous derivation of a homogenized bending-torsion theory for inextensible rods from three-dimensional elasticity, Arch. Ration. Mech. Anal., 206 (2012), pp. 645706, https://doi.org/10.1007/s00205-012-0539-y.

[51] G. NGUEtseng, A general convergence result for a functional related to the theory of homogenization, SIAM J. Math. Anal., 20 (1989), pp. 608-623, https://doi.org/10.1137/0520043.

[52] O. A. OlEINNIK AND V. V. ZhiKOV, On the homogenization of elliptic operators with almostperiodic coefficients, Seminario Mat. e. Fis. di Milano, 52 (1982), pp. 149-166, https: //doi.org/10.1007/BF02925004.

[53] G. C. Papanicolaou and S. Varadhan, Boundary value problems with rapidly oscillating random coefficients, in Random Fields, Vols. I, II (Esztergom, 1979), Colloq. Math. Soc. János Bolyai 27, North-Holland, Amsterdam, 1981, pp. 835-873, http://math.stanford. edu/ papanico/pubftp/pubs_old/pap_vara_79.pdf.

[54] R. Penrose, Pentaplexity: A class of non-periodic tilings of the plane, Math. Intelligencer, 2 (1979), pp. 32-37, https://doi.org/10.1007/BF03024384.

[55] P. M. Santos, A-quasi-convexity with variable coefficients, Proc. Roy. Soc. Edinburgh Sect. A, 134 (2004), pp. 1219-1237, https://doi.org/10.1017/S0308210500003711.

[56] D. Shechtman, I. Blech, D. Gratias, and J. W. Cahn, Metallic phase with long-range orientational order and no translational symmetry, Phys. Rev. Lett., 53 (1984), pp. 19511953, https://doi.org/10.1103/PhysRevLett.53.1951.

[57] D. Shechtman and I. A. Blech, The microstructure of rapidly solidified $\mathrm{Al}_{6} \mathrm{Mn}$, Metal. Trans. A, 16 (1985), pp. 1005-1012, https://doi.org/10.1007/BF02811670.

[58] L. TARTAR, Compensated compactness and applications to partial differential equations, in Nonlinear Analysis and Mechanics: Heriot-Watt Symposium, Vol. IV, Res. Notes in Math. 39, Pitman, Boston, 1979, pp. 136-212.

[59] S. Walter and S. Deloudi, Crystallography of Quasicrystals: Concepts, Methods and Structures, Springer Ser. Mater. Sci. 126, Springer Berlin, 2009, https://doi.org/10.1007/ 978-3-642-01899-2.

Copyright (c) by SIAM. Unauthorized reproduction of this article is prohibited. 
[60] N. Wellander, S. Guenneau, and E. Cherkaev, Homogenization of Quasiperiodic Structures and Two-Scale Cut-and-Projection Convergence, preprint, https://arxiv.org/abs/ 1911.03560, 2019.

[61] M. Xu, X. TEng, AND J. Geng, Effect of cooling rates on solidification and microstructure of rapidly solidified $\mathrm{Mg}_{57} \mathrm{Zn}_{37} \mathrm{Y}_{6}$ quasicrystal alloy, J. Mater. Res., 30 (2015), pp. 3324-3330, https://doi.org/10.1557/jmr.2015.283.

Copyright (c) by SIAM. Unauthorized reproduction of this article is prohibited. 